\title{
NOTES ON FEYNMAN INTEGRALS AND RENORMALIZATION
}

\author{
CHRISTOPH BERGBAUER
}

\begin{abstract}
I review various aspects of Feynman integrals, regularization and renormalization. Following Bloch, I focus on a linear algebraic approach to the Feynman rules, and I try to bring together several renormalization methods found in the literature from a unifying point of view, using resolutions of singularities. In the second part of the paper, I briefly sketch the work of Belkale, Brosnan resp. Bloch, Esnault and Kreimer on the motivic nature of Feynman integrals.
\end{abstract}

\section{CONTENTS}

1. Introduction

2. Feynman graphs and Feynman integrals

3. Regularization and renormalization

4. Motives and residues of Feynman graphs References

\section{INTRODUCTION}

In recent years there has been a growing interest in Feynman graphs and their integrals.

Physicists use Feynman graphs and the associated integrals in order to compute certain experimentally measurable quantities out of quantum field theories. The problem is that there are conceptual difficulties in the definition of interacting quantum field theories in four dimensions. The good thing is that nonetheless the Feynman graph formalism is very successful in the sense that the quantities obtained from it match with the quantities obtained in experiment extremely well. Feynman graphs are interpreted as elements of a perturbation theory, i. e. as an expansion of an (interesting) interacting quantum field theory in the neighborhood of a (simple) free quantum field theory. One therefore hopes that a better understanding of Feynman graphs and their integrals could eventually lead to a better understanding of the true nature of quantum field theories, and contribute to some of the longstanding open questions in the field.

Date: May 20, 2010.

Key words and phrases. Feynman graph, Feynman integral, Feynman rules, regularization, renormalization, subspace arrangement, resolution of singularities, Connes-Kreimer Hopf algebra, matroid, multiple zeta value, motive, period. 
A Feynman graph is simply a finite graph, to which one associates a certain integral: The integrand depends on the quantum field theory in question, but in the simplest case it is just the inverse of a direct product of rank 4 quadratic forms, one for each edge of the graph, restricted to a real linear subspace determined by the topology of the graph.

For a general graph, there is currently no canonical way of solving this integral analytically. However, in this simple case where the integrand is algebraic, one can be convinced to regard the integral as a period of a mixed motive, another notion which is not rigorously defined as of today. All these Feynman periods that have been computed so far, are rational linear combinations of multiple zeta values, which are known to be periods of mixed Tate motives, a simpler, and better understood kind of motives. A stunning theorem of Belkale and Brosnan however indicates that this is possibly a coincidence due to the relatively small number of Feynman periods known today: They showed that in fact any algebraic variety defined over $\mathbb{Z}$ is related to a Feynman graph hypersurface (the Feynman period is one period of the motive of this hypersurface) in a quite obscure way.

The purpose of this paper is to review selected aspects of Feynman graphs, Feynman integrals and renormalization in order to discuss some of the recent work by Bloch, Esnault, Kreimer and others on the motivic nature of these integrals. It is based on public lectures given at the ESI in March 2009, at the DESY and IHES in April and June 2009, and several informal lectures in a local seminar in Mainz in fall and winter 2009. I would like to thank the other participants for their lectures and discussions.

Much of my approach is centered around the notion of renormalization, which seems crucial for a deeper understanding of Quantum Field Theory. No claim of originality is made except for section 3.2 and parts of the surrounding sections, which is a review of my own research with R. Brunetti and D. Kreimer [10], and section 3.6 which contains new results.

This paper is not meant to be a complete and up to date survey by any means. In particular, several recent developments in the area, for example the work of Brown [24, 25], Aluffi and Marcolli [1-3], Doryn and Schnetz [35, 75], and the theory of Connes and Marcolli [32] are not covered here.

Acknowledgements. I thank S. Müller-Stach, R. Brunetti, S. Bloch, M. Kontsevich, P. Brosnan, E. Vogt, C. Lange, A. Usnich, T. Ledwig, F. Brown and especially D. Kreimer for discussion on the subject of this paper. I would like to thank the ESI and the organizers of the spring 2009 program on number theory and physics for hospitality during the month of March 2009, and the IHES for hospitality in January and February 2010. My research is funded by the SFB 45 of the Deutsche Forschungsgemeinschaft. 


\section{Feynman GRAPHS AND FEYNMAN INTEGRALS}

For the purpose of this paper, a Feynman graph is simply a finite connected multigraph where "multi" means that there may be several, parallel edges between vertices. Loops, i. e. edges connecting to the same vertex at both ends, are not allowed in this paper. Roughly, physicists think of edges as virtual particles and of vertices as interactions between the virtual particles corresponding to the adjacent edges.

If one has to consider several types of particles, one has several types (colors, shapes etc.) of edges.

Here is an example of a Feynman graph:

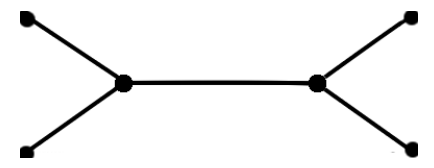

This Feynman graph describes a theoretical process within a scattering experiment: a pair of particles annihilates into a third, intermediate, particle, and this third particle then decays into the two outgoing particles at the right.

This Feynman graph (and the probability amplitude assigned to it) make sense only as a single term in a first order approximation. In order to compute the scattering cross section, one will have to sum over arbitrarily complicated Feynman graphs with four fixed external edges, and in this sum an infinity of graphs with cycles will occur, for example

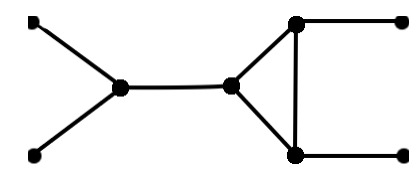

In this paper we will be concerned only with Feynman graphs containing cycles, and I will simply omit the external edges that correspond to the (asymptotic) incoming and outgoing physical particles of a scattering experiment.

I will come back to the physical interpretation in greater detail in section 2.3 .

2.1. Feynman rules. Feynman graphs are not only a nice tool for drawing complex interactions of virtual particles, they also provide a recipe to compute the probability that certain scattering processes occur. The theoretical reason for this will be explained later, but to state it very briefly, a Feynman graph is regarded as a label for a term in a perturbative expansion of this probability amplitude. This term in this expansion is called Feynman integral, but at this point one must be careful with the word integral because of reasons of convergence.

Definition 2.1. An integral is a pair $(A, u)$ where $A$ is an open subset of some $\mathbb{R}^{n}$ or $\mathbb{R}_{\geq 0}^{n}$, and $u$ a distribution in $A \cap\left(\mathbb{R}^{n} \backslash \bigcup H_{i}\right)$ where $H_{i}$ are affine subspaces.

A distribution in $X$ is a continuous linear functional on the space of compactly supported test functions $C_{0}^{\infty}(X)$ with the usual topology. Locally integrable functions (that is, functions integrable on compact subsets) define distributions in an obvious way. Let us denote by $\underline{1}_{A}$ the characteristic function of $A$ in $\mathbb{R}^{n}$. It is 
certainly not a test function unless $A$ is compact, but if $u$ allows (decays rapidly enough at $\infty$ ), then we may evaluate $u$ against $\underline{1}_{A}$. We write $u[f]$ for the distribution applied to the test function $f$. If $u$ is given by a locally integrable function, we may also write $\int u(x) f(x) d x$.

If $u$ is given by a function which is integrable in all of $A$, then $(A, u)$ can be associated with the usual integral $\int_{A} u(x) d x=u\left[\underline{1}_{A}\right]$. Feynman integrals however are very often divergent: This means by definition that $\int_{A} u(x) d x$ is divergent, and this can either result from problems with local integrability at the $H_{i}$ or lack of integrability at $\infty$ away from the $H_{i}$ (if $A$ is unbounded), or both. (A more unified point of view would be to start with a $\mathbb{P}^{n}$ instead of $\mathbb{R}^{n}$ in order to have the divergence at $\infty$ as a divergence at the hyperplane $H_{\infty}$ at $\infty$, but I will not exploit this here).

A basic example for such a divergent integral is the pair $A=\mathbb{R} \backslash\{0\}$ and $u(x)=|x|^{-1}$. The function $u$ is locally integrable inside $A$, hence a distribution in $A$. But neither is it integrable as $|x| \rightarrow \infty$, nor locally integrable at $\{0\}$. We will see in a moment that the divergent Feynman integrals to be defined are higherdimensional generalizations of this example, with an interesting arrangement of the $H_{i}$.

The following approach, which I learned from S. Bloch [14, 15], is quite powerful when one wants to understand the various Feynman rules from a common point of view. It is based on the idea that a Feynman graph first defines a point configuration in some $\mathbb{R}^{n}$, and it is only this point configuration which determines the Feynman integral via the Feynman rules.

Let $\Gamma$ be a Feynman graph with set of edges $E(\Gamma)$ and set of vertices $V(\Gamma)$. A subgraph $\gamma$ has by definition the same vertex set $V(\gamma)=V(\Gamma)$ but $E(\gamma) \subseteq E(\Gamma)$. Impose temporarily an orientation of the edges, such that every edge has an incoming $v_{e, \text { in }}$ and an outgoing vertex $v_{e, \text { out }}$. Since we do not allow loops, the two are different. Set $(v: e)=1$ if $v$ is the outgoing vertex of $e,(v: e)=-1$ if $v$ is the incoming vertex and $e$, and $(v: e)=0$ otherwise. Let $\mathcal{M}=\mathbb{R}^{d}$, where $d \in 2+2 \mathbb{N}$, called space-time, with euclidean metric $|\cdot|$. We will mostly consider the case where $d=4$, but it is useful to see the explicit dependence on $d$ in the formulas.

All the information of $\Gamma$ is encoded in the map

$$
\mathbb{Z}^{E(\Gamma)} \stackrel{\partial}{\rightarrow} \mathbb{Z}^{V(\Gamma)}
$$

sending an edge $e \in E(\Gamma)$ to $\partial(e)=\sum_{v \in V(\Gamma)}(v: e) v=v_{e, \text { out }}-v_{e, \text { in }}$. This is nothing but the chain complex for the oriented simplicial homology of the 1dimensional simplicial complex $\Gamma$, and it is a standard construction to build from this map $\partial$ an exact sequence

$$
0 \rightarrow H_{1}(\Gamma ; \mathbb{Z}) \rightarrow \mathbb{Z}^{E(\Gamma) \stackrel{\partial}{\rightarrow}} \mathbb{Z}^{V(\Gamma)} \rightarrow H_{0}(\Gamma ; \mathbb{Z}) \rightarrow 0 .
$$


Like this one obtains two inclusions of free abelian groups into $\mathbb{Z}^{E(\Gamma)}$ :

$$
i_{\Gamma}: H_{1}(\Gamma ; \mathbb{Z}) \hookrightarrow \mathbb{Z}^{E(\Gamma)}
$$

The second one is obtained by dualizing

$$
j_{\Gamma}: \mathbb{Z}^{V(\Gamma) \vee} / H^{0}(\Gamma ; \mathbb{Z}) \stackrel{\partial^{\vee}}{\hookrightarrow} \mathbb{Z}^{E(\Gamma) \vee} .
$$

Here, and generally whenever a basis is fixed, we can canonically identify free abelian groups with their duals.

All this can be tensored with $\mathbb{R}$, and we get inclusions $i_{\Gamma}, j_{\Gamma}$ of vector spaces into another vector space with a fixed basis. If one then replaces any $\mathbb{R}^{n}$ by $\mathcal{M}^{n}$ and denotes $i_{\Gamma}^{\oplus d}=\left(i_{\Gamma}, \ldots, i_{\Gamma}\right), j_{\Gamma}^{\oplus d}=\left(j_{\Gamma}, \ldots, j_{\Gamma}\right)$, then two types of Feynman integrals $(A, u)$ are defined as follows:

$$
\begin{aligned}
A_{M}=H_{1}(\Gamma ; \mathbb{R})^{d}, & u_{\Gamma}^{M}=\left(i_{\Gamma}^{\oplus d}\right)^{*} u_{0, M}^{\otimes|E(\Gamma)|}, \\
A_{P}=\mathcal{M}^{V(\Gamma) \vee} / H^{0}(\Gamma ; \mathbb{R})^{d}, & u_{\Gamma}^{P}=\left(j_{\Gamma}^{\oplus d}\right)^{*} u_{0, P}^{\otimes|E(\Gamma)|} .
\end{aligned}
$$

The distributions $u_{0, M}, u_{0, P} \in \mathcal{D}^{\prime}(\mathcal{M})$ therein are called momentum space resp. position space propagators. Several examples of propagators and how they are related will be discussed in the next section, but for a first reading

$$
u_{0, M}(p)=\frac{1}{|p|^{2}}, \quad u_{0, P}(x)=\frac{1}{|x|^{d-2}},
$$

inverse powers of a rank $d$ quadratic form. As announced earlier, the pullbacks $\left(i_{\Gamma}^{\oplus d}\right)^{*} u_{0, M}^{\otimes|E(\Gamma)|}$ and $\left(j_{\Gamma}^{\oplus d}\right)^{*} u_{0, M}^{\otimes|E(\Gamma)|}$ are only defined as distributions outside certain affine spaces $H_{i}$, that is for test functions supported on compact subsets which do not meet these $H_{i}$.

The map

$$
\Gamma \mapsto\left(A_{M}, u_{\Gamma}^{M}\right)
$$

is called momentum space Feynman rules, and the map

$$
\Gamma \mapsto\left(A_{P}, u_{\Gamma}^{P}\right)
$$

is called position space Feynman rules.

Usually, in the physics literature, the restriction to the subspace is imposed by multiplying the direct product of propagators with several delta distributions which are interpreted as "momentum conservation" at each vertex in the momentum space picture, and dually "translation invariance" in the position space case.

In position space, it is immediately seen that

$$
u_{\Gamma}^{P}=\left(j_{\Gamma}^{\oplus d}\right)^{*} u_{0, P}^{\otimes|E(\Gamma)|}=\pi_{*} \prod_{e \in E(\Gamma)} u_{0, P}\left(x_{e, \text { out }}-x_{e, \text { in }}\right)
$$


where $\pi_{*}$ means pushforward along the projection $\pi: M^{V(\Gamma) \vee} \rightarrow M^{V(\Gamma) \vee} / H^{0}(\Gamma)^{d}$. [10].

In momentum space, things are a bit more complicated.

Definition 2.2. A connected graph $\Gamma$ is called core if $\operatorname{rk} H_{1}(\Gamma \backslash\{e\})<\operatorname{rk} H_{1}(\Gamma)$ for all $e \in E(\Gamma)$.

By Euler's formula (which follows from the exactness of (1))

$$
\operatorname{rk} H_{1}(\Gamma)-|E(\Gamma)|+|V(\Gamma)|-\operatorname{rk} H_{0}(\Gamma)=0,
$$

it is equivalent for a connected graph $\Gamma$ to be core and to be one-particle-irreducible (1PI), a physicists' notion: $\Gamma$ is one-particle-irreducible if removing an edge does not disconnect $\Gamma$.

Let now $\Gamma$ be connected and core, then

$$
u_{\Gamma}^{M}=\left(i_{\Gamma}^{\oplus d}\right)^{*} u_{0, M}^{\otimes|E(\Gamma)|}=\prod_{e \in E(\Gamma)} u_{0, M}\left(p_{e}\right) \prod_{v \in V(\Gamma)} \delta_{0}\left(\sum_{e \in E(\Gamma)}(v: e) p_{e}\right) .
$$

This is simply because $\operatorname{im} i_{\Gamma}=\operatorname{ker} \partial$, and because for

$$
\partial\left(\sum_{e \in E(\Gamma)} p_{e} e\right)=\sum_{e \in E(\Gamma)} p_{e} \sum_{v}(v: e) v=0
$$

it is necessary that

$$
\sum_{e \in E(\Gamma)}(v: e) p_{e}=0 \text { for all } v \in V(\Gamma) .
$$

(The requirement that $\Gamma$ be core is really needed here because otherwise certain $e \in E(\Gamma)$ would never show up in a cycle, and hence would be missing inside the delta function.)

Moreover, one can define a version of $u_{\Gamma}^{M}$ which depends additionally on external momenta $P_{v} \in \mathcal{M}$, one for each $v \in V(\Gamma)$, up to momentum conservation for each component $\sum_{v \in C} P_{v}=0$ :

$$
U_{\Gamma}^{M}\left(\left\{P_{v}\right\}_{v \in V(\Gamma)}\right)=\prod_{e \in E(\Gamma)} u_{0, M}\left(p_{e}\right) \prod_{v \in V(\Gamma)} \delta_{0}\left(P_{v}+\sum_{e \in E(\Gamma)}(v: e) p_{e}\right) .
$$

By a slight abuse of notation I keep the $P_{v}, v \in V(\Gamma)$, as coordinate vectors for $\mathcal{M}^{|V(\Gamma)|} / H^{0}(\Gamma, \mathbb{R})^{d}=A_{P}$ and identify distributions on $A_{P}$ with distributions on $\mathcal{M}^{|V(\Gamma)|}$ that are multiples of $\prod_{C} \delta_{0}\left(\sum_{v \in C} P_{v}\right)$.

$U_{\Gamma}^{M}$ is now a distribution on a subset of $A_{P} \times A_{M}$, and

$$
\left.U_{\Gamma}^{M}\right|_{P_{v}=0, v \in V(\Gamma)}=u_{\Gamma}^{M} .
$$

The vectors in $P_{v} \in A_{P}$ determine a shift of the linear subspace $A_{M}=H_{1}(\Gamma ; \mathbb{R})^{\oplus d} \hookrightarrow$ $\mathcal{M}^{|E(\Gamma)|}$ to an affine one. Usually all but a few of the $P_{v}$ are set to zero, namely all but those which correspond to the incoming or outgoing particles of an experiment 
(see section 2.3).

The relation between the momentum space and position space distributions is then a Fourier duality. I denote by $\mathcal{F}$ the Fourier transform.

Proposition 2.1. If the basic propagators are Fourier-dual $\left(\mathcal{F} u_{0, P}=u_{0, M}\right)$, as is the case for $u_{0, M}(p)=\frac{1}{|p|^{2}}$ and $u_{0, P}(x)=\frac{1}{|x|^{d-2}}$, then

$$
\left(U_{\Gamma}^{M}\left[\underline{1}_{A_{M}}\right]\right)\left(\left\{P_{v}\right\}\right)=\mathcal{F} u_{\Gamma}^{P}
$$

where only the (internal) momenta of $A_{M}$ are integrated out; and this holds up to convergence issues only, i. e. in the sense of Definition 2.1

For example, the graph

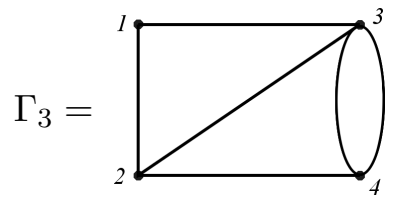

gives rise to

$u_{\Gamma_{3}}^{M}=u_{0, M}^{2}\left(p_{1}\right) u_{0, M}\left(p_{2}\right) u_{0, M}\left(p_{1}+p_{2}\right) u_{0, M}\left(p_{3}\right) u_{0, M}\left(p_{2}+p_{3}\right)$,

$u_{\Gamma_{3}}^{P}=u_{0, P}\left(x_{1}-x_{2}\right) u_{0, P}\left(x_{1}-x_{3}\right) u_{0, P}\left(x_{2}-x_{3}\right) u_{0, P}\left(x_{2}-x_{4}\right) u_{0, P}^{2}\left(x_{3}-x_{4}\right)$,

where $p_{1}^{i}, \ldots, p_{3}^{i}, i=0, \ldots, d-1$ is a basis of coordinates for $A_{M}$ and $x_{1}^{i}, \ldots, x_{4}^{i}$, $i=0, \ldots, d-1$ is a basis of coordinates for $\mathcal{M}^{V\left(\Gamma_{3}\right) \vee}$ (If $\Gamma$ is connected, dividing by $H^{0}(\Gamma ; \mathbb{R})^{d}$ takes care of the joint (diagonal) translations by $\mathcal{M}$ and, as previously, instead of writing distributions on $\mathcal{M}^{V(\Gamma) \vee} / H^{0}(\Gamma ; \mathbb{R})^{d}$, I take the liberty of writing translation-invariant distributions on $\left.\mathcal{M}^{V(\Gamma) \vee}\right)$.

Finally the case of external momenta:

$$
\begin{aligned}
U_{\Gamma_{3}}^{M}\left(P_{1}, P_{2}, 0, P_{4}\right)= & u_{0, M}\left(p_{1}\right) u_{0, M}\left(p_{1}+P_{1}\right) u_{0, M}\left(p_{2}\right) u_{0, M}\left(p_{1}+p_{2}+P_{1}+P_{2}\right) \\
& \times u_{0, M}\left(p_{3}\right) u_{0, M}\left(p_{2}+p_{3}+P_{4}\right) \delta_{0}\left(P_{1}+P_{2}+P_{4}\right) .
\end{aligned}
$$

I set one of the external momenta, $P_{3}$, to zero in order to have a constant number of 4 adjacent (internal and external) momenta at each vertex: $P_{1}$ is the sum of two external momenta at the vertex 1 (See section 2.3 for the reason).

We will come back to the question of the affine subspaces $H_{i}$ where $u_{\Gamma}^{M}$ resp. $u_{\Gamma}^{P}$ is not defined in the section about renormalization.

In general, following [15, Section 2], a configuration is just an inclusion of a vector space $W$ into another vector space $\mathbb{R}^{E}$ with fixed basis $E$ : The dual basis vectors $e^{\vee}, e \in E$ determine linear forms on $W$, and those linear forms (or dually the linear hyperplanes annihilated by them) are the "points" of the configuration in the usual sense. By the above construction, any such configuration, plus the choice of a propagator, defines an integral. 
If the configuration comes from a Feynman graph, the integral is called Feynman integral.

2.2. Parametric representation. Integrals can be rewritten in many ways, using linearity of the integrand, of the domain, change of variables and Stokes' theorem, and possibly a number of other tricks.

For many purposes it will be useful to have a version of the Feynman rules with a domain $A$ which is much lower-dimensional than in the previous section but has boundaries and corners. The first part of the basic trick here is to rewrite the propagator

$$
u_{0}=\int_{0}^{\infty} \exp \left(-a_{e} u_{0}^{-1}\right) d a_{e}
$$

(whenever the choice of propagator allows this inversion; $u_{0}(p)=\frac{1}{|p|^{2}}$ certainly does), introducing a new coordinate $a_{e} \in \mathbb{R}_{\geq 0}$ for each edge $e \in E(\Gamma)$. Like this one has a distribution

$$
\bigotimes_{e \in E(\Gamma)} \exp \left(-a_{e} u_{0}^{-1}\left(p_{e}\right)\right)=\exp \left(-\sum_{e \in E(\Gamma)} a_{e} u_{0}^{-1}\left(p_{e}\right)\right)
$$

in $\left(\mathcal{M} \times \mathbb{R}_{\geq 0}\right)^{|E(\Gamma)|}$. From now on I assume $u_{0}(p)=\frac{1}{|p|^{2}}$. Suppose $i: W \hookrightarrow$ $\mathbb{R}^{|E(\Gamma)|}$ is an inclusion. Once a basis of $W$ is fixed, the linear form $e^{\vee} i$ is a row vector in $W$ and its transpose $\left(e^{\vee} i\right)^{t}$ a column vector in $W$. The product $\left(e^{\vee} i\right)^{t}\left(e^{\vee} i\right)$ is then a $\operatorname{dim} W$-square matrix. Pulling back (4) along an inclusion $i^{\oplus d}: W \hookrightarrow$ $\mathcal{M}^{|E(\Gamma)|}$ (such as $i^{\oplus d}=i_{\Gamma}^{\oplus d}$ or $i^{\oplus d}=j_{\Gamma}^{\oplus d}$ ) means imposing linear relations on the $p_{e}$. These relations can be transposed onto the $a_{e}$ : After integrating gaussian integrals over $W$ (this is the second part of the trick) and a change of variables, one is left with the distribution

$$
u_{\Gamma}^{S}\left(\left\{a_{e}\right\}\right)=\left(\operatorname{det} \sum_{e \in E(\Gamma)} a_{e}\left(e^{\vee} i\right)^{t}\left(e^{\vee} i\right)\right)^{-d / 2}
$$

on $A_{S}=\mathbb{R}_{\geq 0}^{|E(\Gamma)|}$ except certain intersections $H_{i}$ of coordinate hyperplanes $\left\{a_{e}=\right.$ $0\}$. I discarded a multiplicative constant $C_{\Gamma}=(2 \pi)^{d \operatorname{dim} W / 2}$ which does not depend on the topology of the graph.

Suppose that $d=4$. Depending on whether $i=i_{\Gamma}$ or $j_{\Gamma}$ there is a momentum space and a position space version of this trick. The two are dual to each other in the following sense:

$$
\operatorname{det} \sum_{e \in E(\Gamma)} a_{e}\left(e^{\vee} i_{\Gamma}\right)^{t}\left(e^{\vee} i_{\Gamma}\right)=\left(\prod_{e \in E(\Gamma)} a_{e}\right) \operatorname{det} \sum_{e \in E(\Gamma)} a_{e}^{-1}\left(e^{\vee} j_{\Gamma}\right)^{t}\left(e^{\vee} j_{\Gamma}\right)
$$

See [15, Proposition 1.6] for a proof. In this paper, we will only consider the momentum space version, where $i=i_{\Gamma}$. The map

$$
\Gamma \mapsto\left(A_{S}, u_{\Gamma}^{S}\right)
$$


with $i=i_{\Gamma}$ is called Schwinger or parametric Feynman rules. Just as in the previous section, there is also a version with external momenta which I just quote from [14, 16, 47]:

$$
U_{\Gamma}^{S}\left(\left\{a_{e}\right\},\left\{P_{v}\right\}\right)=\frac{\exp \left(-\left(N^{-1} P\right)^{t} P\right)}{\left(\operatorname{det} \sum_{e \in E(\Gamma)} a_{e}\left(e^{\vee} i_{\Gamma}\right)^{t}\left(e^{\vee} i_{\Gamma}\right)\right)^{2}}
$$

where

$$
N=\sum_{e \in E(\Gamma)} a_{e}^{-1}\left(e^{\vee} j_{\Gamma}\right)^{t}\left(e^{\vee} j_{\Gamma}\right),
$$

a $d\left(|V(\Gamma)|-\operatorname{dim} H_{0}(\Gamma ; \mathbb{R})\right)$-square matrix.

The determinant

$$
\Psi_{\Gamma}\left(a_{e}\right)=\operatorname{det} \sum_{e \in E(\Gamma)} a_{e}\left(e^{\vee} i_{\Gamma}\right)^{t}\left(e^{\vee} i_{\Gamma}\right)
$$

is a very special polynomial in the $a_{e}$. It is called first graph polynomial, Kirchhoff polynomial or Symanzik polynomial. It can be rewritten

$$
\Psi_{\Gamma}\left(a_{e}\right)=\sum_{T \text { sf of } \Gamma \notin \notin E(T)} \prod_{e} a_{e}
$$

as a sum over spanning forests $T$ of $\Gamma$ : A spanning forest is a subgraph $E(T) \subseteq$ $E(\Gamma)$ such that the map $\left.\partial\right|_{\mathbb{R}^{E(T)}}: \mathbb{R}^{E(T)} \rightarrow \mathbb{R}^{V(\Gamma)} / H_{0}(\Gamma ; \mathbb{R})$ is an isomorphism; in other words, a subgraph without cycles that has exactly the same components as $\Gamma$. (In the special case where $\Gamma$ is connected, a spanning forest is called a spanning tree and is characterized by being connected as well and having no cycles.)

For the second graph polynomial $\Phi_{\Gamma}$, which is a polynomial in the $a_{e}$ and a quadratic form in the $P_{v}$, let us assume for simplicity that $\Gamma$ is connected. Then

$$
\Phi_{\Gamma}\left(a_{e}, P_{v}\right)=\Psi_{\Gamma} \cdot\left(N^{-1} P\right)^{t} P=\sum_{T \text { st of } \Gamma e_{0} \in E(T)} \sum_{1}^{t} P_{2} a_{e_{0}} \prod_{e \notin E(T)} a_{e}
$$

where $P_{A}=\sum_{v \in C_{A}} P_{v}$ is the sum of momenta in the first connected component $C_{A}$ and $P_{B}=\sum_{v \in C_{B}} P_{v}$ the sum of momenta in the second connected component $C_{B}$ of the graph $E(T) \backslash\left\{e_{0}\right\}$ (which has exactly two components since $T$ is a spanning tree). See [15, 16, 47] for proofs.

Here is a simple example: If

$$
\Gamma_{2}=\bigcup_{2}^{1}
$$


then

$$
\begin{aligned}
& \Psi_{\Gamma_{2}}=a_{1}+a_{2} \\
& \Phi_{\Gamma_{2}}=P_{1}^{2} a_{1} a_{2}
\end{aligned}
$$

and

$$
U_{\Gamma}^{S}=\frac{\exp \left(-P_{1}^{2} \frac{a_{1} a_{2}}{a_{1}+a_{2}}\right)}{\left(a_{1}+a_{2}\right)^{2}} .
$$

All this holds if $u_{0, M}=\frac{1}{|p|^{2}}$. If $u_{0, M}=\frac{1}{|p|^{2}+m^{2}}$ then

$$
U_{\Gamma}^{S}=\left.\exp \left(-m^{2} \sum_{e \in E(\Gamma)} a_{e}\right) U_{\Gamma}^{S}\right|_{m=0}
$$

2.3. The origin of Feynman graphs in physics. Before we continue with a closer analysis of the divergence locus of these Feynman integrals, it will be useful to have at least a basic understanding of why they were introduced in physics. See [28, 33, 42, 45, 54, 72, 86, 87], for a general exposition, and I follow in particular [42,72] in this section. Quantum Field Theory is a theory of particles which obey the basic principles of quantum mechanics and special relativity at the same time. Special relativity is essentially the study of the Poincaré group

$$
\mathcal{P}=\mathbb{R}^{1,3} \rtimes \mathrm{SL}(2, \mathbb{C})
$$

(where $\mathrm{SL}(2, \mathbb{C}) \rightarrow O(1,3)^{+}$is the universal double cover of the identity component $O(1,3)^{+}$of $\left.O(1,3)\right)$. In other words, $\mathcal{P}$ is the double cover of the group of (space- and time-) orientation-preserving isometries of Minkowski space-time $\mathbb{R}^{1,3}$ (I assume $d=4$ in this section).

On the other hand, quantum mechanics always comes with a Hilbert space, a vacuum vector, and operators on the Hilbert space.

By definition, a single particle is then an irreducible unitary representation of $\mathcal{P}$ on some Hilbert space $H_{1}$. Those have been classified by Wigner according to the joint spectrum of $P=\left(P_{0}, \ldots, P_{3}\right)$, the vector of infinitesimal generators of the translations: Its joint spectrum (as a subset of $\mathbb{R}^{1,3}$ ) is either one of the following $\mathrm{SL}(2, \mathbb{C})$-orbits: the hyperboloids (mass shells) $S_{ \pm}(m)=\left\{\left(p^{0}\right)^{2}-\left(p^{1}\right)^{2}-\left(p^{2}\right)^{2}-\right.$ $\left.\left(p^{3}\right)^{2}=m^{2}, p^{0} \gtrless 0\right\} \subset \mathbb{R}^{1,3},(m>0)$, and the forward- and backward lightcones $S_{ \pm}(0) \subset \mathbb{R}^{1,3}(m=0)$. (There are two more degenerate cases, for example $m<0$ which I don't consider further.) This gives a basic distinction between massive $(m>0)$ and massless particles $(m=0)$. For a finer classification, one looks at the stabilizer subgroups $G_{p}$ at $p \in S_{ \pm}(m)$. If $m>0, G_{p} \cong S U(2, \mathbb{C})$, If $m=0$, $G_{p}$ is the double cover of the group of isometries of the euclidean plane. In any case, the $G_{p}$ are pairwise conjugate in $\operatorname{SL}(2, \mathbb{C})$ and

$$
H_{1}=\int_{\oplus} H^{p} d \Omega_{m}(p)
$$


where the $H^{p}$ are pairwise isomorphic and carry an irreducible representation of $G_{p}$. By $d \Omega_{m}$ I denote the unique $\operatorname{SL}(2, \mathbb{C})$-invariant measure on $S_{ \pm}$. The second classifying parameter is then an invariant of the representation of $G_{p}$ on $H^{p}:$ In the case where $m>0$ and $G_{p} \cong S U(2, \mathbb{C})$, one can take the dimension: $H^{p} \cong \mathbb{C}^{2 s+1}$, and $s \in \mathbb{N} / 2$ is called spin. If $m=0, G_{p}$ acts on $\mathbb{C}$ by mapping a rotation by the angle $\phi$ around the origin to $e^{i n \phi} \in \mathbb{C}^{*}$, and $n / 2$ is called helicity (again I dismiss a few cases which are of no physical interest).

In summary, one identifies a single particle of mass $m$ and spin $s$ or helicity $n$ with the Hilbert space

$$
H_{1} \cong L_{2}\left(S_{ \pm}(m), d \Omega_{m}\right) \otimes \mathbb{C}^{2 s+1} \text { resp. } L_{2}\left(S_{ \pm}(0), d \Omega_{0}\right),
$$

and a state of the given particle is an element of the projectivized Hilbert space $\mathbb{P} H_{1}$.

Quantum field theories describe many-particle systems, and particles can be generated and annihilated. A general result in quantum field theory, the SpinStatistics theorem $[62,55]$, tells that systems of particles with integer spin obey Bose (symmetric) statistics while those with half-integer spin obey Fermi (antisymmetric) statistics. We stick to the case of $s=0$, and most of the time even $m=0, n=0$, (which can be considered as a limit $m \rightarrow 0$ of the massive case) in this paper.

The Hilbert space of infinitely many non-interacting particles of the same type, called Fock space, is then

$$
H=\operatorname{Sym}_{1}=\bigoplus_{n=0}^{\infty} \operatorname{Sym}^{n} H_{1}
$$

the symmetric tensor algebra of $H_{1}$ (For fermions, one would use the antisymmetric tensor algebra). $\mathcal{P}$ acts on $H$ in the obvious way, denote the representation by $U$, and $\Omega=1 \in \mathbb{C}=\operatorname{Sym}^{0} H_{1} \subset H$ is called vacuum vector.

Particles are created and annihilated as follows: If $f \in \mathcal{D}\left(\mathbb{R}^{1,3}\right)$ is a test function, then $\hat{f}=\left.\mathcal{F} f\right|_{S_{ \pm}(m)} \in H_{1}$, (the Fourier transform is taken with respect to the Minkowski metric) and

$$
\begin{aligned}
a^{\dagger}[f]: \operatorname{Sym}^{n-1} H_{1} & \rightarrow \operatorname{Sym}^{n} H_{1}: \\
\Phi\left(p_{1}, \ldots, p_{n-1}\right) & \mapsto \sum_{i=1}^{n} \hat{f}\left(p_{i}\right) \Phi\left(p_{1}, \ldots, \widehat{p_{i}}, \ldots, p_{n}\right) \\
a[f]: \operatorname{Sym}^{n+1} H_{1} & \rightarrow \operatorname{Sym}^{n} H_{1}: \\
\Phi\left(p_{1}, \ldots, p_{n+1}\right) & \mapsto \int_{S_{ \pm}(m)} \overline{\hat{f}(p)} \Phi\left(p, p_{1}, \ldots, p_{n}\right) d \Omega_{m}(p),
\end{aligned}
$$


define operator-on- $H$-valued distributions $f \mapsto a^{\dagger}[f], f \mapsto a[f]$ on $\mathbb{R}^{1,3}$. The operator $a^{\dagger}[f]$ creates a particle in the state $\hat{f}$ (i. e. with smeared momentum $\hat{f}$ ), and $a[f]$ annihilates one.

The sum

$$
\phi=a+a^{\dagger}
$$

is called field. It is the quantized version of the classical field, which is a $C^{\infty}$ function on Minkowski space. The field $\phi$ on the other hand is an operator-valued distribution on Minkowski space. It satisfies the Klein-Gordon equation

$$
\left(\square+m^{2}\right) \phi=0
$$

( $\square$ is the Laplacian of $\mathbb{R}^{1,3}$ ) which is the Euler-Lagrange equation for the classical Lagrangian

$$
\mathcal{L}_{0}=\frac{1}{2}\left(\partial_{\mu} \phi\right)^{2}-\frac{1}{2} m^{2} \phi^{2}
$$

The tuple $(H, U, \phi, \Omega)$ and one extra datum which I omit here for simplicity is what is usually referred to as a quantum field theory satisfying the Wightmanaxioms [77]. The axioms require certain $\mathcal{P}$-equivariance, continuity and locality conditions.

The tuple I have constructed (called the free scalar field theory) is a very well understood one because (6) resp. the Lagrangian (7) are very simple indeed. As soon as one attempts to construct a quantum field theory $\left(H_{I}, U_{I}, \phi_{I}, \Omega_{I}\right)$ for an interacting Lagrangian (which looks more like a piece of the Lagrangian of the Standard model) such as

$$
\mathcal{L}_{0}+\mathcal{L}_{I}=\frac{1}{2}\left(\partial_{\mu} \phi_{I}\right)^{2}-\frac{1}{2} m^{2} \phi_{I}^{2}+\lambda \phi_{I}^{n},
$$

( $n \geq 3, \lambda \in \mathbb{R}$ is called coupling constant) one runs into serious trouble. In this rigorous framework the existence and construction of non-trivial interacting quantum field theories in four dimensions is as of today an unsolved problem, although there is an enormous number of important partial results, see for example [74].

However, one can expand quantities of the interacting quantum field theory as a formal power series in $\lambda$ with coefficients quantities of the free field theory, and hope that the series has a positive radius of convergence. This is called the perturbative expansion. In general the power series has radius of convergence 0 , but due to some non-analytic effects which I do not discuss further, the first terms in the expansion do give a very good approximation to the experimentally observed quantities for many important interacting theories (this is the reason why quantum field theories play such a prominent role in the physics of the last 50 years).

I will devote the remainder of this section to a sketch of this perturbative expansion, and how the Feynman integrals introduced in the previous section arise there. 
By Wightman's reconstruction theorem [77], a quantum field theory $\left(H_{I}, U_{I}, \phi_{I}, \Omega_{I}\right)$ is uniquely determined by and can be reconstructed from the Wightman functions (distributions) $w_{n}^{I}=\left\langle\Omega_{I}, \phi_{I}\left(x_{1}\right) \ldots \phi_{I}\left(x_{n}\right) \Omega_{I}\right\rangle$. Similar quantities are the timeordered Wightman functions

$$
t_{n}^{I}=\left\langle\Omega_{I}, T\left(\phi_{I}\left(x_{1}\right) \ldots \phi_{I}\left(x_{n}\right)\right) \Omega_{I}\right\rangle
$$

which appear directly in scattering theory. If one knows all the $t_{n}^{I}$, one can compute all scattering cross-sections. The symbol $T$ denotes time-ordering:

$$
\begin{aligned}
T\left(\psi_{1}\left(x_{1}\right) \psi_{2}\left(x_{2}\right)\right) & =\psi_{1}\left(x_{1}\right) \psi_{2}\left(x_{2}\right) \text { if } x_{1}^{0} \geq x_{2}^{0} \\
& =\psi_{2}\left(x_{2}\right) \psi_{1}\left(x_{1}\right) \text { if } x_{2}^{0}>x_{1}^{0}
\end{aligned}
$$

for operator-valued distributions $\psi_{1}, \psi_{2}$.

For the free field theory, all the $w_{n}$ and $t_{n}$ are well-understood, in particular

$$
\begin{aligned}
t_{2}\left(x_{1}, x_{2}\right) & =\left\langle\Omega, T\left(\phi\left(x_{1}\right) \phi\left(x_{2}\right)\right) \Omega\right\rangle \\
& =\mathcal{F}^{-1} \frac{i}{\left(p^{0}\right)^{2}-\left(p^{1}\right)^{2}-\left(p^{2}\right)^{2}-\left(p^{3}\right)^{2}-m^{2}+i \epsilon}
\end{aligned}
$$

where the Fourier transform is taken with respect to the difference coordinates $x_{1}-x_{2}$ (the $t_{n}$ are translation-invariant). $t_{2}$ is a particular fundamental solution of equation (6) called the propagator. By a technique called Wick rotation, one can go forth and back between Minkowski space $\mathbb{R}^{1,3}$ and euclidean $\mathbb{R}^{4}$ [48, 70], turning Lorentz squares $\left(p^{0}\right)^{2}-\left(p^{1}\right)^{2}-\left(p^{2}\right)^{2}-\left(p^{3}\right)^{2}$ into euclidean squares $-|p|^{2}$, and the Minkowski space propagator $t_{2}$ into the distribution $u_{0, P}=\mathcal{F}^{-1} \frac{1}{|p|^{2}+m^{2}}$ introduced in the previous sections. In the massless case $m=0$, we have $u_{0, P}=$ $u_{0, M}=\frac{1}{|x|^{2}}$ if $d=4$.

From the usual physics axioms for scattering theory and on a purely symbolic level, Gell-Mann's and Low's formula relates the interacting $t_{n}^{I}$ with vacuum expectation values $\langle\Omega, T(\ldots) \Omega\rangle$ of time-ordered products of powers of the free fields (9)

$t_{n}^{I}\left(x_{1}, \ldots, x_{n}\right)=\sum_{k=0}^{\infty} \frac{i^{k}}{k !} \int\left\langle\Omega, T\left(\phi\left(x_{1}\right) \ldots \phi\left(x_{n}\right) \mathcal{L}_{I}^{0}\left(y_{1}\right) \ldots \mathcal{L}_{I}^{0}\left(y_{k}\right)\right) \Omega\right\rangle d^{4} y_{1} \ldots d^{4} y_{k}$

as a formal power series in $\lambda$. I denote $\mathcal{L}_{I}^{0}=\left.\mathcal{L}_{I}\right|_{\phi_{I} \rightarrow \phi}=\lambda \phi^{n}$. (There is a subtle point here in defining powers of $\phi$ as operator-valued distributions. The solution is called Wick powers: In $\phi^{n}=\left(a+a^{\dagger}\right)^{n}$, all monomials containing $a a^{\dagger}$ in this order are discarded.) But now within the free field theory, the $\langle\Omega, T(\ldots) \Omega\rangle$ are well-understood: It follows from the definition of $T, a, a^{\dagger}$ and the Wick powers that $\langle\Omega, T(\ldots) \Omega\rangle$ is a polynomial in the $t_{2}$, more precisely

$$
\left\langle\Omega, T\left(\phi^{n_{1}} \ldots \phi^{n_{k}}\right) \Omega\right\rangle=\sum_{\Gamma} c_{\Gamma} \pi^{*} u_{\Gamma}^{P}
$$


where the sum is over all Feynman graphs $\Gamma$ with $k$ vertices such that the $i$ th vertex has degree $n_{i}$, and where $u_{\Gamma}^{P}$ is defined as in the previous sections, $c_{\Gamma}$ a combinatorial symmetry factor, and $u_{0, P}(x)=t_{2}(x, 0)$ up to a Wick rotation.

If one uses (10) for (9) then one gets Feynman graphs with $n$ external vertices of degree 1 . The external edges, i. e. edges leading to those $n$ vertices, appear simply as tensor factors, and can be omitted (amputated) in a first discussion. Like this we are left with the graphs considered in the previous section.

It follows in particular that only Feynman graphs with vertices of degree $n$ appear from the Lagrangian (8). Note that whereas external physical particles are always on-shell (i. e. their momentum supported on $S_{ \pm}$), the internal virtual particles are integrated over all of momentum space in the Gell-Mann-Low formula.

In summary, the perturbative expansion of an interacting quantum field theory (whose existence let alone construction in the sense of the Wightman axioms is an unsolved problem) provides an power series approximation in the coupling constant to the bona fide interacting functions $t_{n}^{I}$. The coefficients are sums of Feynman integrals which are composed of elements of the free theory only.

\section{REGULARIZATION AND RENORMALIZATION}

The Feynman integrals introduced so far are generally divergent integrals. At first sight it seems to be a disturbing feature of a quantum field theory that it produces divergent integrals in the course of calculations, but a closer look reveals that this impression is wrong: it is only a naive misinterpretation of perturbation theory that makes us think that way.

Key to this is the insight that single Feynman graphs are really about virtual particles, and their parameters, for example their masses, have no real physical meaning. They have to be renormalized. Like this the divergences are compensated by so-called counterterms in the Lagrangian of the theory which provide some kind of dynamical contribution to those parameters [28]. I will not make further use of this physical interpretation but only consider mathematical aspects. If the divergences can be compensated by adjusting only a finite number of parameters in the Lagrangian (i. e. by leaving the form of the Lagrangian invariant and not adding an infinity of new terms to it) the theory is called renormalizable.

An important and somehow nontrivial, but fortunately solved [19, 29, 30, 38, 46. 53, 89], problem is to find a way to organize this correspondence between removing divergences and compensating counterterms in the Lagrangian for arbitrarily complicated graphs. Since the terms in the Lagrangian are local terms, that is polynomials in the field and its derivatives, a necessary criterion for this is the socalled locality of counterterms: If one has a way of removing divergences such that the correction terms are local ones, then this is a good indication that they fit into 
the Lagrangian in the first place.

Regularization on the other hand is the physics term used for a variety of methods of writing the divergent integral or integrand as the limit of a holomorphic family of convergent integrals or integrands, say over a punctured disk. Sometimes also the integrand is fixed, and the domain of integration varies holomorphically say over the punctured disk. We will see a number of such regularizations in the remainder of this paper.

3.1. Position space. In position space, the renormalization problem has been known for a long time to be an extension problem of distributions [19, 38]. This follows already from our description in section 2 , but it will be useful to have a closer look at the problem. Recall the position space Feynman distribution

$$
u_{\Gamma}^{P}=\left(j_{\Gamma}^{\oplus d}\right)^{*} u_{0, P}^{\otimes|E(\Gamma)|}
$$

is defined only as a distribution on $A_{P}=\mathcal{M}^{|V(\Gamma)| \vee} / H^{0}(\Gamma ; \mathbb{R})^{\oplus d}$ minus certain affine (in this case even linear) subspaces. Suppose for example

$$
\Gamma_{2}=\bigcap_{2}^{1}
$$

with $u_{\Gamma_{2}}^{P}=\frac{1}{|x|^{2 d-4}}$. If $f$ is a non-negative test function supported in a ball $N=$ $\{|x| \leq \epsilon\}$ around 0 .

$$
u_{\Gamma_{2}}^{P}[f]=\int_{N} f(x) u_{\Gamma_{2}}^{P}(x) d x \geq \min _{x \in N} f(x) \int d \Omega \int_{0}^{\epsilon} \frac{d r^{d-1}}{r^{2 d-4}} .
$$

If $d-1-(2 d-4) \leq-1$, that is $d \geq 4$, the integral will be divergent at 0 and $u_{\Gamma_{2}}^{P}$ not defined on test functions supported at 0 . This is the very nature of ultraviolet (i. e. short-distance) divergences. On the other hand, divergences as some positionspace coordinates go to $\infty$, are called infrared (long-distance) divergences. We will be concerned with ultraviolet divergences in this paper.

For simplicity we restrict ourselves to graphs with at most logarithmic divergences throughout the rest of the paper, that is $d \operatorname{rk} H_{1}(\gamma) \geq 2|E(\gamma)|$ for all subgraphs $E(\gamma) \subseteq E(\Gamma)$. A subgraph $\gamma$ where equality holds is called divergent. A detailed power-counting analysis, carried out in [10] shows that $u_{\Gamma}^{P}$ is only defined as a distribution inside

$$
A_{P}^{\circ}=A_{P} \backslash \bigcup_{\substack{E(\gamma) \subseteq E(\Gamma) \\ d \mathrm{rk} H_{1}(\gamma)=2|E(\gamma)|}} \bigcap_{e \in E(\gamma)} \pi D_{e}
$$

where $D_{e}=\left\{x_{e, \text { out }}-x_{e, \text { in }}=0\right\}$. The singular support (the locus where $u_{\Gamma}^{P}$ is not smooth) is

$$
\operatorname{sing} \operatorname{supp} u_{\Gamma}^{P}=A_{P}^{\circ} \cap \bigcup_{e \in E(\Gamma)} \pi D_{e} .
$$


An extension of $u_{\Gamma}^{P}$ from $A_{P}^{\circ}$ to $A_{P}$ is called a renormalization provided it satisfies certain consistency conditions to be discussed later.

In the traditional literature, which dates back to a central paper of Epstein and Glaser [38], an extension of $u_{\Gamma}^{P}$ from $A_{P}^{\circ}$ to all of $A_{P}$ was obtained inductively, by starting with the case of two vertices, and embedding the solution (extension) for this case into the three, four, etc. vertex case using a partition of unity. Like this, in each step only one extension onto a single point, say 0 , is necessary, a wellunderstood problem with a finite-dimensional degree of freedom: Two extensions differ by a distribution supported at this point 0 , and the difference is therefore, by an elementary consideration, of the form $\sum_{|\alpha| \leq n} c_{\alpha} \partial^{\alpha} \delta_{0}$ with $c_{\alpha} \in \mathbb{C}$. Some of these parameters $c_{\alpha}$ are fixed by physical requirements such as probability conservation, Lorentz and gauge invariance, and more generally the requirement that certain differential equations be satisfied by the extended distributions. But even after these constants are fixed, there are degrees of freedom left, and various groups act on the space of possible extensions, which are collectively called renormalization group. For the at most logarithmic graphs considered in this paper, $n=0$ and only one constant $c_{0}$ needs to be fixed in each step.

3.2. Resolution of singularities. The singularities, divergences and extensions (renormalizations) of the Feynman distribution $u_{\Gamma}^{P}$ are best understood using a resolution of singularities [10]. The Fulton-MacPherson compactification [43] introduced in a quantum field theory context by Kontsevich [49, 51] and Axelrod and Singer [6] serves as a universal smooth model where all position space Feynman distributions can be renormalized. In [10], a graph-specific De Concini-Procesi Wonderful model [34] was used, in order to elaborate the striking match between De Concini's and Procesi's notions of building set, nested set and notions found in Quantum Field Theory. No matter which smooth model is chosen, one disposes of a smooth manifold $Y$ and a proper surjective map, in fact a composition of blowups,

$$
\beta: Y \rightarrow A_{P}
$$

which is a diffeomorphism on $\beta^{-1}\left(A_{P}^{\circ}\right)$ but $\beta^{-1}\left(A_{P} \backslash A_{P}^{\circ}\right)$ is (the real locus of) a divisor with normal crossings.

Instead of the nonorientable smooth manifold $Y$ one can also find an orientable manifold with corners $Y^{\prime}$ and $\beta$ a composition of real spherical blowups as in [6]. In my pictures, the blowups are spherical because they are easier to draw, but in the text they are projective.

Here is an example: If $\Gamma_{3}$ is again the graph

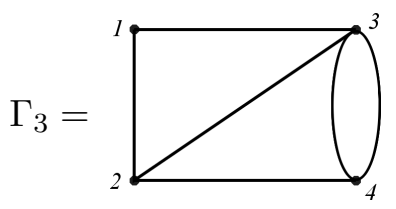


and $d=4$ then by (11) the locus where there are nonintegrable singularities is

$$
D_{1234} \subset D_{234} \subset D_{34}
$$

where $D_{1234}=D_{12} \cap D_{13} \cap D_{14}, D_{234}=D_{23} \cap D_{24}$. In $A_{P}, \pi D_{1234}$ is a point, $\pi D_{234}$ is 4-dimensional and $\pi D_{24}$ is 8 -dimensional. Blowing up something means replacing it by its projectivized normal bundle. The map $\beta$ is composed of three maps

$$
Y=Y_{34} \stackrel{\beta_{3}}{\rightarrow} Y_{234} \stackrel{\beta_{2}}{\rightarrow} Y_{1234} \stackrel{\beta_{1}}{\rightarrow} A_{P}
$$

where $\beta_{1}$ blows up $D_{1234}, \beta_{2}$ blows up the strict transform of $D_{234}$, and $\beta_{3}$ blows up the strict transform of $D_{34}$.

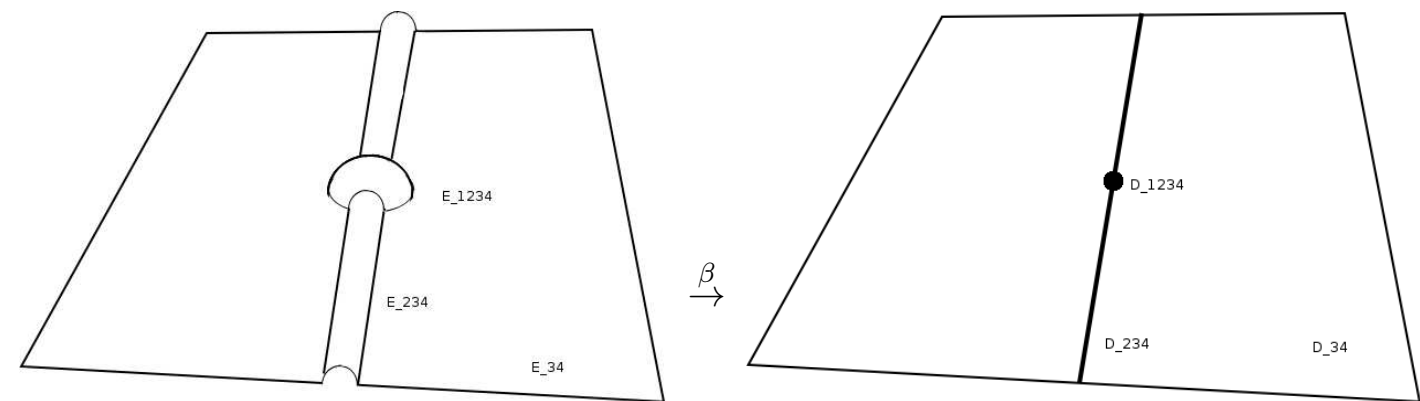

Now $u_{\Gamma_{3}}^{P}$ can be pulled back along $\beta$ (because of lack of orientability of $Y$, it will become a distribution density). In a clever choice of local coordinates, for example

$$
\begin{aligned}
y_{1}^{0} & =x_{1}^{0}-x_{2}^{0} \\
y_{2}^{0} & =\left(x_{2}^{0}-x_{3}^{0}\right) /\left(x_{1}^{0}-x_{2}^{0}\right) \\
y_{3}^{0} & =\left(x_{3}^{0}-x_{4}^{0}\right) /\left(x_{2}^{0}-x_{3}^{0}\right) \\
y_{1}^{i} & =\left(x_{1}^{i}-x_{2}^{i}\right) /\left(x_{1}^{0}-x_{2}^{0}\right) \\
y_{2}^{i} & =\left(x_{2}^{i}-x_{3}^{i}\right) /\left(x_{2}^{0}-x_{3}^{0}\right) \\
y_{3}^{i} & =\left(x_{3}^{i}-x_{4}^{i}\right) /\left(x_{3}^{0}-x_{4}^{0}\right)
\end{aligned}
$$

one has

$$
w_{\Gamma_{3}}^{P}=\beta^{*} u_{\Gamma_{3}}^{P}=\frac{f_{\Gamma_{3}}^{P}}{\left|y_{1}^{0} y_{2}^{0} y_{3}^{0}\right|}
$$

where $f_{\Gamma_{3}}^{P}$ is a locally integrable density which is even $C^{\infty}$ in the coordinates $y_{1}^{0}, y_{2}^{0}, y_{3}^{0}$. The divergence is therefore isolated in the denominator, and only in three directions: $y_{1}^{0}, y_{2}^{0}$ and $y_{3}^{0}$. The first is the local coordinate transversal to the exceptional divisor $\mathcal{E}_{1234}$ of the blowup of $D_{1234}$, the second transversal to the exceptional divisor $\mathcal{E}_{234}$ of the blowup of $D_{234}$, and the third transversal to the exceptional divisor $\mathcal{E}_{34}$ of the blowup of $D_{34}$ (the difference between $\mathcal{E}_{34}$ and $D_{34}$ is not seen in the picture because of dimensional reasons). 
For a general graph $\Gamma$, the total exceptional divisor $\mathcal{E}=\beta^{-1}\left(A_{P} \backslash A_{P}^{\circ}\right)$ has normal crossings and the irreducible components $\mathcal{E}_{\gamma}$ are indexed by connected divergent (consequently core) irreducible subgraphs $\gamma$. Moreover,

$$
\mathcal{E}_{\gamma_{1}} \cap \ldots \cap \mathcal{E}_{\gamma_{k}} \neq \emptyset \Longleftrightarrow \text { the } \gamma_{i} \text { are nested }
$$

where nested means each pair is either disjoint or one contained in the other. See [10] for the general result and more details.

Inspired by old papers of Atiyah [5], Bernstein and Gelfand [12] we used $\left(u_{\Gamma}^{P}\right)^{s}$, where $s$ in a complex number in a punctured neighborhood of 1 , as a regularization [10]. Similarly, since the propagator $u_{0, P}(x)=\frac{1}{|x|^{d-2}}$ depends on the dimension, one can also consider $u_{\Gamma}^{P}$ with $d$ in a punctured complex neighborhood of 4 as a regularization but I will not pursue this here.

Definition 3.1. A connected graph $\Gamma$ is called primitive if

$$
d \operatorname{rk} H_{1}(\gamma)=2|E(\gamma)| \Longleftrightarrow E(\gamma)=E(\Gamma) .
$$

for all subgraphs $E(\gamma) \subseteq E(\Gamma)$.

For a primitive graph $\Gamma_{p}$, only the single point $0 \in A_{P}$ needs to be blown up, and the pullback along $\beta$ yields in suitable local coordinates $\left(y_{1}^{0}=x_{1}^{0}-x_{2}^{0}\right.$, $y_{i}^{j}=\left(x_{i}^{j}-x_{i+1}^{j}\right) /\left(x_{1}^{0}-x_{2}^{0}\right)$ otherwise $)$

$$
\beta^{*} u_{\Gamma_{p}}^{P}=\frac{f_{\Gamma_{p}}}{\left|y_{1}^{0}\right|}
$$

where $f_{\Gamma_{p}}$ is a locally integrable distribution density constant in $y_{1}^{0}$-direction. Let $d_{\Gamma}=d\left(\left|V\left(\Gamma_{p}\right)\right|-1\right)$. Consequently

$$
\beta^{*}\left(u_{\Gamma_{p}}^{P}\right)^{s}=\frac{f_{\Gamma_{p}}^{s}}{\left|y_{1}^{0}\right|^{d_{\Gamma_{p}} s-\left(d_{\Gamma_{p}}-1\right)}}
$$

It is well-known that the distribution-valued function $\frac{1}{|x|^{s}}$ can be analytically in a punctured neighborhood of $s=1$, with a simple pole at $s=1$. The residue of this pole is $\delta_{0}$ :

$$
\frac{1}{|x|^{s}}=\frac{\delta_{0}}{s-1}+|x|_{\text {fin }}^{s}, \quad|x|_{\text {fin }}^{s}[f]=\int_{-1}^{1}|x|^{s}(f(x)-f(0)) d x+\int_{\mathbb{R} \backslash[-1,1]}|x|^{s} f(x) d x .
$$

This implies that the residue at $s=1$ of $\beta^{*}\left(u_{\Gamma_{p}}^{P}\right)^{s}$ is a density supported at the exceptional divisor (which is given in these coordinates by $y_{0}=0$, and integrating this density against the constant function $\underline{1}_{Y}$ gives what is called the residue of the graph $\Gamma_{p}$

$$
\operatorname{res}_{P} \Gamma_{p}=\operatorname{res}_{s=1} \beta^{*}\left(u_{\Gamma_{p}}^{P}\right)^{s}\left[\underline{1}_{Y}\right]=-\frac{2}{d_{\Gamma_{p}}} \int_{\mathcal{E}} f_{\Gamma_{p}}
$$

(The exceptional divisor can actually be oriented in such a way that $f_{\Gamma_{p}}$ is a degree $\left(d_{\Gamma_{p}}-1\right)$ differential form). 
Let us now come back to the case of $\Gamma_{3}$ which is not primitive but has a nested set of three divergent subgraphs. Raising (13) to a power $s$ results in a pole at $s=1$ of order 3. The Laurent coefficient $a_{-3}$ of $(s-1)^{-3}$ is supported on

$$
\mathcal{E}_{1234} \cap \mathcal{E}_{234} \cap \mathcal{E}_{34},
$$

for this is the set given in local coordinates by $y_{1}^{0}=y_{2}^{0}=y_{3}^{0}=0$. Similarly, the coefficient of $(s-1)^{-2}$ is supported on

$$
\left(\mathcal{E}_{1234} \cap \mathcal{E}_{234}\right) \cup\left(\mathcal{E}_{1234} \cap \mathcal{E}_{34}\right) \cup\left(\mathcal{E}_{234} \cap \mathcal{E}_{34}\right)
$$

and the coefficient of $(s-1)^{-1}$ on

$$
\mathcal{E}_{1234} \cup \mathcal{E}_{234} \cup \mathcal{E}_{34} .
$$

(The non-negative part of the Laurent series is supported everywhere on $Y$ ). Write $|d y|=\left|d y_{1}^{0} \ldots d y_{3}^{3}\right|$. In order to compute the coefficient $a_{-3}$, one needs to integrate $f_{\Gamma_{3}}$, restricted to the subspace $y_{1}^{0}=y_{2}^{0}=y_{3}^{0}=0$ :

$$
\begin{aligned}
f_{\Gamma_{3}}= & \frac{|d y|}{\left(1+\underline{y}_{1}^{2}\right)\left(1+\underline{y}_{2}^{2}\right)\left(1+\underline{y}_{3}^{2}\right)} \\
& \times \frac{1}{\left.\left(\left(1+y_{2}^{0}\right)^{2}+\left(\underline{y}_{1}+y_{2}^{0} \underline{y}_{2}\right)^{2}\right)\left(\left(1+y_{3}^{0}\right)^{2}+\left(\underline{y}_{2}+y_{3}^{0} \underline{y}_{3}\right)^{2}\right)\right)}
\end{aligned}
$$

where $\underline{y}_{i}$ denotes the 3 -vector $\left(y_{i}^{1}, y_{i}^{2}, y_{i}^{3}\right)$. Consequently

$$
\left.f_{\Gamma_{3}}\right|_{y_{1}^{0}=y_{2}^{0}=y_{3}^{0}=0}=\frac{|d y|}{\left(1+\underline{y}_{1}^{2}\right)^{2}\left(1+\underline{y}_{2}^{2}\right)^{2}\left(1+\underline{y}_{3}^{2}\right)^{2}}=f_{\Gamma_{1}}^{\otimes 3}
$$

where $\Gamma_{1}$ is the primitive graph with two vertices and two parallel edges joining them:

$$
\Gamma_{1}=\bigcap_{2}^{1}
$$

The chart where (13) holds covers actually everything of $Y_{P}$ up to a set of measure zero where there are no additional divergences. It suffices therefore to integrate in these coordinates only. Several charts must be taken into account however when there are more than one maximal nested set. In conclusion,

$$
a_{-3}\left[\underline{1}_{Y}\right]=\left(\operatorname{res}_{P} \Gamma_{1}\right)^{3},
$$

a special case of a theorem in [10] relating pole coefficients of $\beta^{*}\left(u_{\Gamma}^{P}\right)^{s}$ to residues of graphs obtained from $\Gamma$ by contraction of divergent subgraphs.

But the ultimate reason to introduce the resolution of singularities in the first place is: In order to obtain an extension (renormalization) of $u_{\Gamma}^{P}$, one can now 
simply remove the simple pole at $s=1$ along each component of the exceptional divisor:

$$
\begin{aligned}
w_{\Gamma_{3}}^{P} & =\frac{f_{\Gamma_{3}}}{\left|y_{1}^{0} y_{2}^{0} y_{3}^{0}\right|} \\
\left(w_{\Gamma_{3}}^{P}\right)_{R} & =\frac{f_{\Gamma_{3}}}{\left|y_{1}^{0}\right|_{\text {fin }}\left|y_{2}^{0}\right|_{\text {fin }}\left|y_{3}^{0}\right|_{\text {fin }}} .
\end{aligned}
$$

The second distribution $\left(w_{\Gamma_{3}}^{P}\right)_{R}$ is defined on all of $Y$, and consequently $\beta_{*}\left(w_{\Gamma_{3}}^{P}\right)_{R}$ on all of $A_{P}$. It agrees with $u_{\Gamma_{3}}^{P}$ on test functions having support in $A_{P}^{\circ}$ and is therefore an extension. The difference between $w_{\Gamma_{3}}^{P}$ and $\left(w_{\Gamma_{3}}^{P}\right)_{R}$ is a distribution supported on the exceptional divisor which gives rise to a candidate for a counterterm in the Lagrangian.

I call this renormalization scheme local minimal subtraction, because locally, along each component of the exceptional divisor, the simple pole is removed in a "minimal way", changing only the principal part of the Laurent series. See [10] for a proof that this results in local counterterms, a necessary condition for the extension to be a physically consistent one.

3.3. Momentum space. In momentum space, the bad definition of the position space Feynman distribution at certain diagonals $\bigcap D_{e}$ is translated by a Fourier transform into ill-defined (divergent) integrals with divergences at certain strata at infinity. For example, the position space integral $\left(\mathcal{M}, u_{\Gamma_{1}}^{P}=u_{0, P}^{2}\right)$ in $d=4$ dimensions for the graph $\Gamma_{1}$ (see (14) ) has a divergence at 0 (which is the image $\pi D_{12}$ of the diagonal). A formal Fourier transform would turn the pointwise product $u_{0, P}^{2}$ into a convolution product

$$
\left(\mathcal{F} u_{0, P}^{2}\right)(P)=\int u_{0, M}(p) u_{0, M}(p-P) d^{4} p .
$$

In fact the right hand side is exactly $U_{\Gamma_{1}}^{M}(P)\left[\underline{1}_{A_{\Gamma_{1}}}\right]$ in agreement with Proposition 2.1. It does not converge at $\infty$. (In order to see this we actually only need $\left.U_{\Gamma_{1}}^{M}\right|_{P=0}=u_{\Gamma_{1}}^{M}$, not the dependence upon external momenta).

On the other hand, the infrared singularities are to be found at affine subspaces in momentum space. Of course the program sketched in the previous section can be applied to the momentum space Feynman distribution as well: A resolution of singularities for the relevant strata at infinity can be found, and the pullback of the momentum space Feynman distribution can be extended onto all the irreducible components of the exceptional divisor. But I want to use this section in order to sketch another, algebraic, approach to the momentum space renormalization problem, which is due to Connes and Kreimer [29, 30, 53].

Assume $U_{\Gamma}^{M}\left[\underline{1}_{A_{M}}\right]$ varies holomorphically with $d$ in a punctured disk around $d=4$. Physicists call this dimensional regularization [32,39]: any integral $\int d^{4} p u(p) d p$ is replaced by a $d$-dimensional integral $\int d^{d} p u(p) d p$. Like this we can consider 
$U_{\Gamma}^{M}$ as a distribution on all of $A_{P} \times A_{M}$ with values in $\mathcal{R}=\mathbb{C}\left[\left[(d-4)^{-1},(d-4)\right]\right]$, the field of Laurent series in $d-4$. If $U_{\Gamma}^{M}[f]$ is not convergent in $d=4$ dimensions, then there will be a pole at $d=4$.

Let now $\sigma_{\Gamma} \in \mathcal{D}^{\prime}\left(A_{P}\right)$ be a distribution with compact support. Since the distribution $U_{\Gamma}^{M}$ is smooth in the $P_{v}$, we can actually integrate it against the distribution $\sigma_{\Gamma}$ (For example, if $\sigma_{\Gamma}=\delta_{0}\left(\left|P_{v_{1}}\right|^{2}-E_{1}\right) \otimes \ldots \otimes \delta_{0}\left(\left|P_{v_{n}}^{2}\right|-E_{n}\right)$ then this amounts simply to evaluating $U_{\Gamma}^{M}$ at the subspaces $\left|P_{v_{1}}\right|^{2}=E_{1}, \ldots,\left|P_{v_{2}}\right|^{2}=E_{n}$ ). In any case we have a map

$$
\phi:\left(\Gamma, \sigma_{\Gamma}\right) \mapsto U_{\Gamma}^{M}\left[\underline{1}_{A_{M}} \otimes \sigma_{\Gamma}\right] \in \mathcal{R}
$$

sending pairs to Laurent series. Let now $\mathcal{H}$ be the polynomial algebra over $\mathbb{C}$ generated by isomorphism classes of connected core divergent graphs $\Gamma$ of a given renormalizable quantum field theory. Define a coproduct $\Delta$ by

$$
\Delta(\Gamma)=1 \otimes \Gamma+\Gamma \otimes 1+\sum_{\substack{\gamma_{1} \sqcup \ldots \sqcup \gamma_{k} \subsetneq \Gamma \\ \text { conn. core div. }}} \gamma_{1} \cdots \gamma_{k} \otimes \Gamma / /\left(\gamma_{1} \sqcup \ldots \sqcup \gamma_{k}\right) .
$$

The notation $\Gamma / / \gamma$ means that any connected component of $\gamma$ inside $\Gamma$ is contracted to a (separate) vertex. By standard constructions [29], $\mathcal{H}$ becomes a Hopf algebra, called Connes-Kreimer Hopf algebra. Denote the antipode by $S$. Let now $\mathcal{H}_{\sigma}$ be the corresponding Hopf algebra of pairs $\left(\Gamma, \sigma_{\Gamma}\right)$ (In order to define this Hopf algebra of pairs, one needs the extra condition that $\sigma_{\Gamma}$ vanishes on all vertices that have no external edges, a standard assumption if one considers only graphs of a fixed renormalizable theory).

The map $\phi: \mathcal{H}_{\sigma} \rightarrow \mathcal{R}$ is a homomorphism of unital $\mathbb{C}$-algebras. The space of these maps $\mathcal{H}_{\sigma} \rightarrow \mathcal{R}$ is a group with the convolution product $\phi_{1} \star \phi_{2}=m\left(\phi_{1} \otimes\right.$ $\left.\phi_{2}\right) \Delta$. On $\mathcal{R}$, there is the linear projection

$$
R:(d-4)^{n} \mapsto \begin{cases}0 & \text { if } n \geq 0 \\ (d-4)^{n} & \text { if } n<0\end{cases}
$$

onto the principal part.

Theorem 3.1 (Connes, Kreimer). The renormalized Feynman integral $\left.\phi_{R}\left(\Gamma, \sigma_{\Gamma}\right)\right|_{d=4}$ and the counterterm $S_{R}^{\phi}\left(\Gamma, \sigma_{\Gamma}\right)$ are given as follows. I denote $\underline{\Gamma}$ for the pair $\left(\Gamma, \sigma_{\Gamma}\right)$ :

$$
\begin{aligned}
& S_{R}^{\phi}(\underline{\Gamma})=-R\left(\phi(\underline{\Gamma})+\sum_{\substack{\gamma=\gamma_{1} \sqcup \ldots \sqcup \gamma_{k} \subsetneq \Gamma \\
\text { conn. core div. }}} S_{R}^{\phi}(\underline{\gamma}) \phi(\underline{\Gamma} / / \underline{\gamma})\right) \\
& \phi_{R}(\underline{\Gamma})=(1-R)\left(\phi(\underline{\Gamma})+\sum_{\substack{\gamma=\gamma_{1} \sqcup \ldots \sqcup \gamma_{k} \subsetneq \Gamma \\
\text { conn. core div. }}} S_{R}^{\phi}(\underline{\gamma}) \phi(\underline{\Gamma} / / \underline{\gamma})\right)
\end{aligned}
$$


These expressions are assembled from the formula for the antipode and the convolution product. Combinatorially, the Hopf algebra encodes the BPHZ recursion [46] and Zimmermann's forest formula [89]. The theorem can be interpreted as a Birkhoff decomposition of the character $\phi$ into $\phi_{-}=S_{R}^{\phi}$ and $\phi_{+}=\phi_{R}$ [30].

The renormalization scheme described here is what I call global minimal subtraction, because in the target field $\mathcal{R}$, when all local information has been integrated out, the map $1-R$ removes only the entire principal part at $d=4$. This coincides with the renormalization scheme described in [28].

In the case of $m=0$ and zero-momentum transfer (all but two external momenta set to 0 ) one knows that at $d=4$

$$
\phi_{R}(\Gamma)=\sum_{n=0}^{N} p_{n}(\Gamma)\left(\log |P|^{2} / \mu^{2}\right)^{n}, \quad p_{n}(\Gamma) \in \mathbb{R}
$$

where $\mu$ is an energy scale, and the $\sigma_{\Gamma}$ can be dropped for convenience. Let us now do our standard example

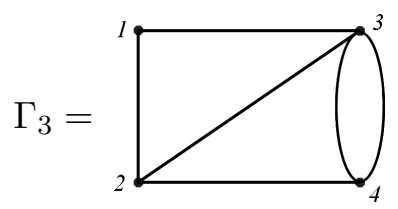

using the Hopf algebra. We interpret $\Gamma_{3}$ as a graph in $\phi^{4}$ theory, so we think of two external edges at the first vertex, one at the second, and one at the fourth. Recall the momentum space Feynman rules (3) for $\Gamma_{3}$. Let $P_{2}=0$ and write $P=P_{1}=-P_{4}$ such that $P_{1}$ is the sum of the two external momenta entering at the first vertex. Then

$$
\phi\left(\Gamma_{3}\right)=\int \frac{d^{d} p_{1} d^{d} p_{2} d^{d} p_{3}}{p_{1}^{2}\left(p_{1}+P\right)^{2} p_{2}^{2}\left(p_{1}+p_{2}+P\right)^{2} p_{3}^{2}\left(p_{2}+p_{3}-P\right)^{2}} \in \mathcal{R} .
$$

This integral can be evaluated as a Laurent series in $d=4$ using standard techniques [28]. It has a pole of order 3 at $d=4$, and one might think of simply taking $(1-R) \phi\left(\Gamma_{3}\right)$ as a renormalized value, for this kills the principal part, and the limit at $d=4$ may be taken. But the resulting counterterms would not be local ones, and the renormalization would be physically inconsistent. The benefit of the Hopf algebra approach is that the necessary correction terms are provided right away:

Let again $\gamma_{1}$ be the full subgraph with vertices 3 and 4 , and $\gamma_{2}$ the full subgraph with vertices 2,3 and 4 . Then

$$
\begin{aligned}
\phi_{R}\left(\Gamma_{3}\right)= & (1-R)\left(\phi\left(\Gamma_{3}\right)-\left(R \phi\left(\gamma_{2}\right)\right) \phi\left(\Gamma_{3} / / \gamma_{2}\right)+\right. \\
& \left.+R\left(\left(R \phi\left(\gamma_{1}\right)\right) \phi\left(\gamma_{2} / / \gamma_{1}\right)\right) \phi\left(\Gamma_{3} / / \gamma_{2}\right)\right) .
\end{aligned}
$$

Observe that, as a coincidental property of our example, $\Gamma_{3} / / \gamma_{2} \cong \gamma_{2} / / \gamma_{1} \cong \gamma_{1}$ (compare this with (15), (23)). 
The Hopf algebra approach to renormalization has brought up a number of surprising connections to other fields, see for example [30,31, 37, 41, 59, 64, 67, 79, 80]. Other developments starting from the Connes-Kreimer theory can be found in [32]. Kreimer and van Suijlekom have shown that gauge and other symmetries are compatible with the Hopf algebra structure [55, 61, 78, 84, 85].

A sketch how the combinatorics of the Hopf algebra relate to the resolution of singularities in the previous section and to position space renormalization can be found in [10], see also section 3.6 .

3.4. Parametric representation. In the parametric representation introduced in section 2.2 the divergences can be found at certain intersections of the coordinate hyperplanes $A_{e}=\left\{a_{e}=0\right\}$. This is in fact one of the very reasons why the parametric representation was introduced: Consider for example the divergent integral $\left(\mathbb{R}^{4}, u_{0, M}^{2}\right)$, with $u_{0, M}=\frac{1}{|p|^{2}}$,

$$
\int \frac{d^{4} p}{|p|^{4}}=\iint_{0}^{\infty} \int_{0}^{\infty} \exp \left(-a_{1}|p|^{2}-a_{2}|p|^{2}\right) d a_{1} d a_{2} d^{4} p
$$

in the sense of Definition 2.1 (In this section, instead of $(A, u)$ I will simply write $\int_{A} u(x) d x$.) The integral at the left hand side is divergent both at 0 and at $\infty$. But splitting it into the two parts at the right, and interchanging the $d^{4} p$ with the $d a_{1} d a_{2}$ integrations leaves a gaussian integral

$$
\int \exp \left(-\frac{c}{2}|p|^{2}\right) d^{d} p=(2 \pi / c)^{d / 2}
$$

which is convergent, but at the expense of getting $\left(a_{1}+a_{2}\right)^{2}$ in the denominator: The integral

$$
\int_{0}^{\infty} \int_{0}^{\infty} \frac{d a_{1} d a_{2}}{\left(a_{1}+a_{2}\right)^{2}}
$$

has a logarithmic singularity at 0 and at $\infty$. This can be seen by blowing up the origin in $\mathbb{R}_{\geq 0}^{2}$, and pulling back:

$$
\int_{0}^{\infty} \int_{0}^{\infty} \frac{d b_{1} d b_{2}}{b_{1}\left(1+b_{2}\right)^{2}}
$$

In other words, the trick with the parametric parameterization (called Schwinger trick in [15]), does not get rid of any divergences. It just moves them into another, lower-dimensional space.

Again it is useful to have a resolution of singularities in order to separate the various singularities and divergences of a graph along irreducible components of a divisor with normal crossings. The most obvious and efficient such resolution is given in [15, 16]:

Let $\Gamma$ be core. For a subgraph $E(\gamma) \subseteq E(\Gamma)$, let

$$
L_{\gamma}=\cap_{e \in E(\gamma)} A_{e}=\left\{a_{e}=0, e \in E(\gamma)\right\}
$$


a linear subspace. Set $\mathcal{L}_{\text {core }}=\left\{L_{\gamma}: \gamma\right.$ is a core subgraph of $\left.\Gamma\right\}$, and

$$
\begin{aligned}
\mathcal{L}_{0} & =\left\{\text { minimal element of } \mathcal{L}_{\text {core }}\right\}=\{0\} \\
\mathcal{L}_{n+1} & =\left\{\text { minimal elements of } \mathcal{L}_{\text {core }} \backslash \bigsqcup_{i=0}^{n} \mathcal{L}_{i}\right\}
\end{aligned}
$$

This partition of $\mathcal{L}_{\text {core }}$ is made in such a way that (see [16, Proposition 3.1]) a sequence of blowups

$$
\gamma: Z_{S} \rightarrow \ldots \rightarrow A_{S}
$$

is possible which starts by blowing up $\mathcal{L}_{0}$ and then successively the strict transforms of the elements of $\mathcal{L}_{1}, \mathcal{L}_{2}, \ldots$ This ends up with $Z_{S}$ a manifold with corners. The map $\gamma$ is of course defined not only as a map onto $A_{S}=\mathbb{R}_{\geq 0}^{|E(\Gamma)|}$ but as a birational map $\gamma: \mathcal{Z}_{S} \rightarrow \mathbb{C}^{|E(\Gamma)|}$, with $\mathcal{Z}_{S}$ a smooth complex variety. The total exceptional divisor $\mathcal{E}$ has normal crossings, and one component $\mathcal{E}_{L}$ for each $L \in \mathcal{L}_{\text {core }}$. (In the language of section 3.2, $\mathcal{L}_{\text {core }}$ is the building set). Moreover,

$$
\mathcal{E}_{L_{1}} \cap \ldots \cap \mathcal{E}_{L_{k}} \neq 0 \Longleftrightarrow \text { the } L_{i} \text { are totally ordered by inclusion. }
$$

Since the coordinate divisor $\left\{a_{e}=0\right.$ for some $\left.e \in E(\Gamma)\right\}$ has already normal crossings by definition, the purpose of these blowups is really only to pull out into codimension 1 all the intersections where there are possibly singularities or divergences, and to separate the integrable singularities of the integrand from this set as much as possible.

Note that in the parametric situation where the domain of integration is the manifold with corners $\mathbb{R}_{\geq 0}^{|E(\Gamma)|}$, the blowups do not introduce an orientation issue on the real locus.

For the example graph $\Gamma_{3}$ of the previous sections (see (12)),

$$
u_{\Gamma_{3}}^{S}=\frac{d a_{1} \ldots d a_{6}}{\left(\left(a_{1}+a_{2}\right)\left(\left(a_{3}+a_{4}\right)\left(a_{5}+a_{6}\right)+a_{5} a_{6}\right)+a_{3} a_{4} a_{5}+a_{3} a_{4} a_{6}+a_{3} a_{5} a_{6}\right)^{d / 2}}
$$

we examine the pullback of $u_{\Gamma_{3}}^{S}$ onto $Z_{S}$. There are various core subgraphs to consider, but it is easily seen, in complete analogy with (11), that the divergences are located only at $L_{\Gamma_{3}}, L_{\gamma_{2}}$ and $L_{\gamma_{1}}$ where $\gamma_{1}$ is the full subgraph with vertices 3 and 4 , and $\gamma_{2}$ the full subgraph with vertices 2,3 and 4 . In order to see the divergences in $Z_{S}$, it therefore suffices to look in a chart where $\mathcal{E}_{L_{\Gamma_{3}}}, \mathcal{E}_{L_{\gamma_{2}}}$ and $\mathcal{E}_{L_{\gamma_{1}}}$ intersect. In such a chart, given by coordinates $b_{1}=a_{1}, b_{2}=a_{2} / a_{1}, b_{3}=a_{3} / a_{1}$, $b_{4}=a_{4} / a_{1}, b_{5}=a_{5} / a_{3}, b_{6}=a_{6} / a_{5}$, we have

$$
\gamma^{*} u_{\Gamma_{3}}^{S}=\frac{d b_{1} \ldots d b_{6}}{b_{1} b_{3} b_{5}\left(\left(1+b_{2}\right)\left(\left(1+b_{6}\right)\left(1+b_{4}\right)+b_{5} b_{6}\right)+b_{3}\left(b_{5} b_{6}+b_{4} b_{6}+b_{4}\right)\right)^{d / 2}}
$$

Now we are in a very similar position as in the previous section. If $\Gamma_{p}$ is a primitive graph, then there is only the origin $0 \in A_{S}$ which needs to be blown up in order to isolate the divergence. Since $u_{\Gamma_{3}}^{S}$ depends explicitly on $d$ in the exponent, let us 
use $d$ as an analytic regulator. One finds, using for example coordinates $b_{1}=a_{1}$, $b_{i}=a_{i} / a_{1}, i \neq 1$, in a neighborhood of $d=4$,

$$
\gamma^{*} u_{\Gamma_{p}}^{S}(d)=\left(\frac{\delta_{0}\left(b_{1}\right)}{d-4}+\text { finite }\right) g_{\Gamma_{p}}
$$

with $g_{\Gamma_{p}} \in L_{l o c}^{1}$. (If one wants even a regular $g_{\Gamma_{p}}$ one needs to perform the remaining blowups in (20).) Then we define

$$
\operatorname{res}_{S} \Gamma_{p}=\left(\operatorname{res}_{d=4} \gamma^{*} u_{\Gamma_{p}}^{S}(d)\right)[\underline{1}]=\int_{b_{1}=0, b_{i} \geq 0} g_{\Gamma_{p}}=\int_{\sigma} \frac{\Omega}{\Psi_{\Gamma_{p}}^{2}}
$$

where $\sigma=\left\{a_{i} \geq 0\right\} \subset \mathbb{P}^{|E(\Gamma)|-1}(\mathbb{R})$ and $\Omega=\sum_{n=1}^{|E(\Gamma)|}(-1)^{n} a_{n} d a_{1} \wedge \ldots \wedge \widehat{d a_{n}} \wedge$ $\ldots \wedge d a_{|E(\Gamma)|}$. The last integral at the right is a projective integral, meaning that the $a_{i}$ are interpreted as homogeneous coordinates of $\mathbb{P}^{|E(\Gamma)|-1}$. By choosing affine coordinates $b_{i}$, one finds that it is identical with the integral of $g_{\Gamma_{p}}$ over the exceptional divisor intersected with the total inverse image of $A_{S}$.

Coming back to the non-primitive graph $\Gamma_{3}$ (see (21)) we find in complete analogy with section 3.2, that

$$
u_{\Gamma_{3}}^{S}(d)=\sum_{n \geq-3}^{\infty} c_{n}(d-4)^{n}
$$

in a neighborhood of $d=4$, and

$$
c_{-3}\left[\underline{1}_{A_{S}}\right]=\left(\operatorname{res}_{S} \Gamma_{1}\right)^{3}
$$

which is easily seen by sending $b_{1}, b_{3}, b_{5}$ to 0 in (21): $\left.g_{\Gamma_{3}}\right|_{b_{1}=b_{3}=b_{5}=0}=g_{\Gamma_{1}}^{\otimes 3}$.

Similarly, one can translate the results of section 3.2] and [10] into this setting and obtain a renormalization (extension of $u_{\Gamma}^{S}$ ) by removing the simple pole along each component of the irreducible divisor. In section 4.5 a different, motivic renormalization scheme for the parametric representation will be studied, following [16].

3.5. Dyson-Schwinger equations. Up to now we have only considered single Feynman graphs, with internal edges interpreted as virtual particles, and parameters such as the mass subjected to renormalization. Another approach is to start with the full physical particles from the beginning, that is, with the non-perturbative objects. Implicit equations satisfied by the physical particles (full propagators) and the physical interactions (full vertices) are called Dyson-Schwinger equations. The equations can be imposed in a Hopf algebra of Feynman graphs [11, 23, 57, 58, 88] and turn into systems of integral equations when Feynman rules are applied.

For general configurations of external momenta, Dyson-Schwinger equations are extremely hard to solve. But if one sets all but two external momenta to 0 , a situation called zero-momentum transfer (see (19)), then the problem simplifies considerably. 
In [60], an example of a linear Dyson-Schwinger equation is given which can be solved nonperturbatively by a very simple Ansatz. More difficult non-linear Dyson-Schwinger equations, and finally systems of Dyson-Schwinger equations as above, are studied in [62, 63, 82, 83], see also [40, 56, 88].

3.6. Remarks on minimal subtraction. I come back at this point to the difference between what I call local (section 3.2) and global (section 3.3) minimal subtraction, which, I think, is an important one.

I tried to emphasize in the exposition of the previous sections that the key concepts of renormalization are largely independent of whether momentum space, position space, or parametric space Feynman rules are used. This is immediately seen in the Connes-Kreimer Hopf algebra framework where a graph $\Gamma$ and some external information $\sigma_{\Gamma}$ are sent directly to a Laurent series in $d-4$. For this we don't get to see and don't need to know if the integral has been computed in momentum, position, or parametric space. They all produce the same number (or rather Laurent series), provided the same regularization is chosen for all three of them.

In position space, where people traditionally like to work with distributions as long as possible and integrate them against a test function only at the very end (or even against the constant function $\underline{1}$, the adiabatic limit), one is tempted to define the Feynman rules as a map into a space of distribution-valued Laurent series, as we have done it in [10]. But one has to be aware that this space of distribution-valued Laurent series does not necessarily qualify as a replacement for the ring $\mathcal{R}$ in section 3.3 if one looks for a new Birkhoff decomposition. In general, many questions and misconceptions that I have encountered in this area can be traced back to the decision at which moment one integrates, and minimal subtraction seems to be a good example for this.

Let me now give a detailed comparison of what happens in local and global minimal subtraction, respectively. Assume for example the massless graph in 4 dimensions

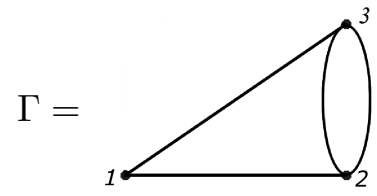

Clearly $\Gamma$ itself and the full subgraph $\gamma$ on the vertices 2 and 3 are logarithmically divergent. No matter which kind of Feynman rules we use, assume there is a regularized Feynman distribution $u_{\Gamma}(\epsilon)$ varying holomorphically in a punctured disk around $\epsilon=0$, with a finite order pole at $\epsilon=0$. Assume after resolution of singularities that the regularized Feynman distribution, pulled back onto the smooth model, has a simple pole supported on the component $\mathcal{E}_{\Gamma}$ of the total exceptional divisor (for the superficial divergence), and another on the component $\mathcal{E}_{\gamma}$ (for the subdivergence). Let $\mathcal{E}_{\Gamma}=\left\{y_{\Gamma}=0\right\}$ and $\mathcal{E}_{\gamma}=\left\{y_{\gamma}=0\right\}$ in local coordinates 
$y_{\Gamma}, y_{\gamma}, y_{3}, \ldots, y_{n}$

$$
u_{\Gamma}(\epsilon)=\left(\frac{\delta_{0}\left(y_{\Gamma}\right)}{\epsilon}+\left|y_{\Gamma}\right|_{\text {fin }}(\epsilon)\right)\left(\frac{\delta_{0}\left(y_{\gamma}\right)}{\epsilon}+\left|y_{\gamma}\right|_{f i n}(\epsilon)\right) f_{\Gamma}(\epsilon)
$$

where $f_{\Gamma}$ is locally integrable and smooth in $y_{\Gamma}$ and $y_{\gamma}$, such that in particular $f_{\Gamma}(\epsilon)$ is holomorphic in $\epsilon$. There is accordingly a second order pole supported at $\mathcal{E}_{\Gamma} \cap \mathcal{E}_{\gamma}$. We know from [10], as was also sketched in section 3.2] that the leading coefficient of this second order pole is a product of delta functions restricting it to $\mathcal{E}_{\Gamma} \cap \mathcal{E}_{\gamma}$ times the residue of $\gamma$ times the residue of $\Gamma / / \gamma$.

Consequently, integrating $u_{\Gamma}(\epsilon)$ against a fixed function $\chi$ (for a first reading take $\chi=\underline{1}$ but in the massless case, one has to worry about infared divergences) provides a Laurent series

$$
u_{\Gamma}(\epsilon)[\chi]=a_{-2} \epsilon^{-2}+a_{-1} \epsilon^{-1}+a_{0} \epsilon^{0}+\ldots
$$

Since $\gamma$ and $\Gamma / / \gamma$ are primitive,

$$
\begin{aligned}
u_{\gamma}(\epsilon)[\chi] & =b_{-1} \epsilon^{-1}+b_{0} \epsilon^{0}+b_{1} \epsilon^{1}+\ldots \\
u_{\Gamma / / \gamma}(\epsilon)[\chi] & =c_{-1} \epsilon^{-1}+c_{0} \epsilon^{0}+b_{1} \epsilon^{1}+\ldots
\end{aligned}
$$

We know from the previous remarks that $a_{-2}=\operatorname{res}(\gamma) \operatorname{res}(\Gamma / / \gamma)=b_{-1} c_{-1}$ and similarly $a_{-1}=b_{-1} c_{0}+g$ where I don't want to specify $g$.

Let me now compare local and global minimal subtraction at this example. Local minimal subtraction is defined on distribution-valued Laurent series, but global minimal subtraction only on $\mathbb{C}$-valued Laurent series. Therefore we need to integrate everything out before comparing. I start with local minimal subtraction (LMS). In order to get from (24) to

$$
\left(u_{\Gamma}\right)_{R, \mathrm{LMS}}(\epsilon)=\left|y_{\Gamma}\right|_{\text {fin }}(\epsilon)\left|y_{\gamma}\right|_{\text {fin }}(\epsilon) f_{\Gamma}(\epsilon)
$$

one has to subtract three terms from (24):

$$
\begin{aligned}
R_{\mathrm{LMS}}^{\Gamma} u_{\Gamma}(\epsilon) & =\frac{\delta_{0}\left(y_{\Gamma}\right)}{\epsilon}\left(\frac{\delta_{0}\left(y_{\gamma}\right)}{\epsilon}+\left|y_{\gamma}\right|_{\text {fin }}(\epsilon)\right) f_{\Gamma}(\epsilon) \\
R_{\mathrm{LMS}}^{\gamma, \Gamma / / \gamma} u_{\Gamma}(\epsilon) & =\left(\frac{\delta_{0}\left(y_{\Gamma}\right)}{\epsilon}+\left|y_{\Gamma}\right|_{\text {fin }}(\epsilon)\right) \frac{\delta_{0}\left(y_{\gamma}\right)}{\epsilon} f_{\Gamma}(\epsilon) \\
-R R_{\mathrm{LMS}}^{\gamma, \Gamma / / \gamma} u_{\Gamma}(\epsilon) & =-\frac{\delta_{0}\left(y_{\Gamma}\right)}{\epsilon} \frac{\delta_{0}\left(y_{\gamma}\right)}{\epsilon} f_{\Gamma}(\epsilon)
\end{aligned}
$$

The first term cleans the pole supported on $\mathcal{E}_{\Gamma}$, such that $u_{\Gamma}-R_{\mathrm{LMS}}^{\Gamma} u_{\Gamma}$ has only a simple pole supported on $\mathcal{E}_{\gamma}$ left. On the other hand, $u_{\Gamma}-R_{\mathrm{LMS}}^{\gamma, \Gamma / / \gamma} u_{\Gamma}$ has only a simple pole supported on $\mathcal{E}_{\Gamma}$ left, and the third term is a correction term supported on $\mathcal{E}_{\gamma} \cap \mathcal{E}_{\Gamma}$ accounting for what has been subtracted twice. In summary,

$$
\left(u_{\Gamma}\right)_{R, \mathrm{LMS}}(\epsilon)=u_{\Gamma}(\epsilon)-R_{\mathrm{LMS}}^{\Gamma} u_{\Gamma}(\epsilon)-R_{\mathrm{LMS}}^{\gamma, \Gamma / / \gamma} u_{\Gamma}(\epsilon)+R R_{\mathrm{LMS}}^{\gamma, \Gamma / / \gamma} u_{\Gamma}(\epsilon)
$$

is the result of local minimal subtraction. 
Let us now integrate out (26).

$$
\begin{aligned}
u_{\Gamma}(\epsilon)[\chi] & =a_{-2} \epsilon^{-2}+a_{-1} \epsilon^{-1}+a_{0} \epsilon^{0}+\ldots \\
R_{\mathrm{LMS}}^{\Gamma} u_{\Gamma}(\epsilon)[\chi] & =a_{-2} \epsilon^{-2}+g \epsilon^{-1}+h \epsilon^{0}+\ldots \\
R_{\mathrm{LMS}}^{\gamma, \Gamma / \gamma} u_{\Gamma}(\epsilon)[\chi] & =a_{-2} \epsilon^{-2}+b_{-1} c_{0} \epsilon^{-1}+b_{-1} c_{1} \epsilon^{0}+\ldots \\
-R R_{\mathrm{LMS}}^{\gamma, \Gamma / \gamma} u_{\Gamma}(\epsilon)[\chi] & =a_{-2} \epsilon^{-2}
\end{aligned}
$$

These equations follow from (24), and I don't want to specify $h$. Consequently

$$
\left(u_{\Gamma}\right)_{R, \mathrm{LMS}}(\epsilon)[\chi]=a_{0}-b_{-1} c_{1}-h \text { as } \epsilon \rightarrow 0 .
$$

In global minimal subtraction (GMS), where $R_{\mathrm{GMS}}=R$ as in (18), something different happens.

$$
\begin{aligned}
R_{\mathrm{GMS}}\left(u_{\Gamma}(\epsilon)[\chi]\right) & =a_{-2} \epsilon^{-2}+a_{-1} \epsilon^{-1} \\
\left(R_{\mathrm{GMS}} u_{\gamma}(\epsilon)[\chi]\right) u_{\Gamma / / \gamma}(\epsilon)[\chi] & =b_{-1} c_{-1} \epsilon^{-2}+b_{-1} c_{0} \epsilon^{-1}+b_{-1} c_{1} \epsilon^{0}+\ldots \\
\left.-R_{\mathrm{GMS}}\left(R_{\mathrm{GMS}} u_{\gamma}(\epsilon)[\chi]\right) u_{\Gamma / / \gamma}(\epsilon)[\chi]\right) & =b_{-1} c_{-1} \epsilon^{-2}+b_{-1} c_{0} \epsilon^{-1}
\end{aligned}
$$

The first subtraction $u_{\Gamma}[\chi]-R_{\mathrm{GMS}}\left(u_{\Gamma}[\chi]\right)$ removes the poles everywhere, also the one supported on $\mathcal{E}_{\gamma}$ which has nothing to do with the superficial divergence. The third and fourth term restore the locality of counterterms. We have

$$
\left(u_{\Gamma}\right)_{R, \mathrm{GMS}}(\epsilon)[\chi]=a_{0}-b_{-1} c_{1} \text { as } \epsilon \rightarrow 0 .
$$

In summary: Unless $h=0$, local and global minimal subtraction differ by a finite renormalization. Moreover, although there is a one-to-one-correspondence between terms to be subtracted in LMS and GMS, the values of those single terms do not agree. It seems to me that GMS is a quite clever but somehow exceptional trick of defining the subtraction operator $R$ on $\mathbb{C}$-valued Laurent series where all the geometric information (i. e. where the pole is supported) has been forgotten.

In [10] it is shown how to relate, for a general graph $\Gamma$, the combinatorics of the total exceptional divisor of the resolution of singularities to the Connes-Kreimer Hopf algebra of Feynman graphs, such that the example presented here is a special case of a more general result. A similar analysis applies to other local renormalization prescriptions, called subtraction at fixed conditions in [10], as well.

\section{Motives AND RESIDUES OF FEYNMAN GRAPHS}

4.1. Motives, Hodge Realization and Periods. Much of the present interest in Feynman integrals is due to the more or less obvious fact that there is something motivic about them. In order to understand and appreciate this, one obviously needs to have an idea of what a motive is. I am not an expert in this area and will not even attempt to provide much background to the notion of motive. See [4] for an often cited introduction to the subject, which I follow closely in the beginning of this section. 
The theory of motives is a means to unify the various cohomology theories known for algebraic varieties $X$ over a number field $k$. Such cohomology theories include the algebraic de Rham and the Betti cohomology, but there are many others. The algebraic de Rham cohomology $H_{d R}^{\bullet}(X)$ is defined over the ground field $k$, and Betti cohomology $H_{B}^{\bullet}(X ; \mathbb{Q})$ is the singular cohomology of $X(\mathbb{C})$ with rational coefficients.

A motive of a variety is supposed to be a piece of a universal cohomology, such that all the usual cohomology theories (functors from varieties to graded vector spaces) factor through the category of motives. A particular cohomology theory is then called a realization. For example, the combination of de Rham and Betti cohomology, giving rise to a Hodge structure, is called Hodge realization.

The theory of motives is not complete yet. Only for the simplest kind of algebraic varieties, smooth projective ones, a category of motives with the desired properties has been constructed. These motives are called pure. For general, i. e. singular or non-projective varieties, the theory is conjectural in the sense that only a triangulated category as a candidate for the derived category of the category of these motives, called mixed motives exists.

Let $X$ be a smooth variety over $\mathbb{Q}$. Let $H_{d R}^{\bullet}(X)$ denote the algebraic de Rham cohomology of $X$, a graded $\mathbb{Q}$-vector space, and $H_{B}^{\bullet}(X ; \mathbb{Q})$ the rational Betti cohomology (singular cohomology of the complex manifold $X(\mathbb{C})$ with rational coefficients), a graded $\mathbb{Q}$-vector space. A period of $X$ is by definition a matrix element of the comparison isomorphism (integration)

$$
H_{d R}^{\bullet}(X) \otimes_{\mathbb{Q}} \mathbb{C} \cong H_{B}^{\bullet}(X ; \mathbb{Q}) \otimes_{\mathbb{Q}} \mathbb{C}
$$

for a suitable choice of basis. A period is therefore in particular an integral of an algebraic differential form over a topological cycle on $X(\mathbb{C})$. A standard example is the case of an elliptic curve $X$ defined by the equation $y^{2}=x(x-1)(x-\lambda)$, $\lambda \in \mathbb{Q} \backslash\{0,1\}$. A basis element of $H_{d R}^{1}(X)$ is the 1-form $\omega=\frac{d x}{2 y}$ and and a basis of the singular cohomology $H_{B}^{1}(X)$ is given by the duals of two circles around the cut between 0 and 1 resp. the cut between 1 and $\infty$. Integrating $\omega$ against these cycles gives the generators of the period lattice of $X$.

Similarly, matrix elements of a comparison isomorphism between relative cohomologies of pairs $(X, A)$ are called relative periods. Many examples considered below will be relative periods.

4.2. Multiple zeta values, mixed Tate motives and the work of Belkale and Brosnan. Let $\Gamma$ be a primitive Feynman graph. I assume $d=4$ and $m=0$. Recall the graph polynomial

$$
\Psi_{\Gamma}=\sum_{T \text { st of } \Gamma} \prod_{e \notin E(T)} a_{e} \in \mathbb{Z}\left[a_{e}: e \in E(\Gamma)\right]
$$


from (5). The sum is over the spanning trees of $\Gamma$. Following [15], we have a closer look at the parametric residue

$$
\operatorname{res}_{S} \Gamma=\int_{\sigma} \frac{\Omega}{\Psi_{\Gamma}^{2}}
$$

introduced in (22). Let $X_{\Gamma}=\left\{\Psi_{\Gamma}=0\right\} \subset \mathbb{P}^{|E(\Gamma)|-1}$ and $C X_{\Gamma}=\left\{\Psi_{\Gamma}=\right.$ $0\} \subset \mathbb{A}^{|E(\Gamma)|}$ its affine cone. $X_{\Gamma}$ resp. $C X_{\Gamma}$ are called projective resp. affine graph hypersurface. The chain of integration is $\sigma=\left\{a_{e} \geq 0\right\} \subseteq \mathbb{P}^{|E(\Gamma)|-1}(\mathbb{R})$, and $\Omega=\sum(-1)^{n} a_{n} d a_{1} \wedge \ldots \wedge \widehat{d a_{n}} \wedge \ldots \wedge d a_{|E(\Gamma)|}$.

The residue $\operatorname{res}_{S} \Gamma$ already looks like a relative period, since $\sigma$ has its boundary contained in the coordinate divisor $\Delta=\bigcup_{e \in E(\Gamma)}\left\{a_{e}=0\right\}$, and the differential form $\frac{\Omega}{\Psi_{\Gamma}^{2}}$ is algebraic (i. e. regular) in $\mathbb{P}^{|E(\Gamma)|-1} \backslash X_{\Gamma}$. But in general $X_{\Gamma} \cap \Delta$ is quite big, and $\frac{\Omega}{\Psi_{\Gamma}^{2}} \notin H_{d R}^{|E(\Gamma)|-1}\left(\mathbb{P}^{|E(\Gamma)|-1} \backslash X_{\Gamma}, \Delta \backslash\left(X_{\Gamma} \cap \Delta\right)\right)$.

The solution is of course to work in the blowup $\mathcal{Z}_{S}$ of section 3.4 where things are separated. Let $\mathcal{P}_{S}$ be the variety obtained from $\mathbb{P}^{|E(\Gamma)|-1}$ by regarding all elements of the $\mathcal{L}_{n}(n \geq 1)$ in section 3.4 as subspaces of $\mathbb{P}^{|E(\Gamma)|-1}$ and starting the blowup sequence at $n=1$ instead of $n=0$.

In [15, 16] it is shown that $\mathcal{P}_{S}$ has the desired properties: the strict transform of $X_{\Gamma}$ does not meet the strict transform of $\sigma$. Like this, $\operatorname{res}_{S} \Gamma$ is a relative period of the pair

$$
\left(\mathcal{P}_{S} \backslash Y_{\Gamma}, B \backslash\left(B \cap Y_{\Gamma}\right)\right)
$$

where $Y_{\Gamma}$ is the strict transform of $X_{\Gamma}$, and $B$ the total transform of the coordinate divisor $\Delta$.

We call $\operatorname{res}_{S} \Gamma$ a Feynman period of $\Gamma$.

An empirical observation due to Broadhurst and Kreimer [21,22] was that all Feynman periods computed so far are rational linear combinations of multiple zeta values.

A multiple zeta value of depth $k$ and weight $s=s_{1}+\ldots+s_{k}$ is a real number defined as follows:

$$
\zeta\left(s_{1}, \ldots, s_{k}\right)=\sum_{1 \leq n_{k}<\ldots<n_{1}} \frac{1}{n_{1}^{s_{1}} \ldots n_{k}^{s_{k}}}
$$

where $s_{1} \geq 2$ and $s_{2}, \ldots, s_{k} \geq 1$. For $k=1$ one obtains the values of the Riemann zeta function at integer arguments $\geq 2$, whence the name. 
By an observation due to Euler and Kontsevich, multiple zeta values can be written as iterated integrals

$$
\zeta\left(s_{1}, \ldots, s_{k}\right)=\int_{0<t_{s}<\ldots<t_{1}<1} w_{s_{1}} \wedge \ldots \wedge w_{s_{k}}
$$

where

$$
w_{s}(t)=\left(\frac{d t}{t}\right)^{\wedge(s-1)} \wedge \frac{d t}{1-t}
$$

and therefore qualify already as naive periods, as defined in [52].

But in order to understand multiple zeta values as (relative) periods of the cohomology of something, one needs to go one step further and introduce the moduli space $\mathcal{M}_{0, s+3}$ of genus 0 curves with $s+3$ distinct marked points, and its DeligneMumford compactification $\overline{\mathcal{M}}_{0, s+3}$.

Indeed, starting from the iterated integral representation, $\zeta\left(s_{1}, \ldots, s_{k}\right)$ can be shown to be a relative period of a pair

$$
\left(\overline{\mathcal{M}}_{0, s+3} \backslash A, B \backslash(A \cap B)\right)
$$

with $A$ and $B$ suitable divisors which have no common irreducible component. These pairs have mixed Tate motives, a special (and relatively simple and wellunderstood) kind of mixed motives. This is a result of Goncharov and Manin [44]. Brown showed that conversely every such relative period of $\overline{\mathcal{M}}_{0, s+3}$ is a rational linear combination of multiple zeta values [26].

Let us now come back to the Feynman periods. Even up to now, not a single example of a Feynman period is known which is not a rational linear combination of multiple zeta values. Moreover, these multiple zeta values do not arise randomly, but there are already certain patterns visible. For examples of such patterns, see [15, 21, 22, 76].

Motivated by an (informal) conjecture of Kontsevich [50], Belkale and Brosnan investigated the motives associated to Feynman graph hypersurfaces. Kontsevich's conjecture did not state directly that all Feynman periods be multiple zeta values, but that the function

$$
q \mapsto\left|C X_{\Gamma}\left(\mathbb{F}_{q}\right)\right|
$$

be a polynomial in $q$ for all $\Gamma$. Using another conjecture about motives, a nonpolynomial counting function for the number of points of $C X_{\Gamma}$ over $\mathbb{F}_{q}$ would imply that $C X_{\Gamma}$ has a period which is not in the $\mathbb{Q}$-span of multiple zeta values. For example, an elliptic curve is known to have a non-polynomial point counting function.

Belkale and Brosnan came to the surprising result that Kontsevich's conjecture is false [7], and that Feynman graph hypersurfaces have the most general motives one can think of. 
4.3. Matroids and Mnëv's theorem. One key idea in Belkale's and Brosnan's proof was to study more general schemes defined by matroids:

Definition 4.1. Let $E$ be a finite set and $I \subseteq 2^{E}$. The pair $M=(E, I)$ is called matroid if

(1) $\emptyset \in I$,

(2) $A_{1} \subseteq A_{2}, A_{2} \in I \Longrightarrow A_{1} \in I$,

(3) $A_{1}, A_{2} \in I,\left|A_{2}\right|>\left|A_{1}\right| \Longrightarrow$ there is an $x \in A_{2} \backslash A_{2} \cap A_{1}$ such that $A_{1} \cup\{x\} \in I$.

The number $\operatorname{rk} M=\max _{A \in I}|A|$ is called rank of $M$.

The subsets $A \in I$ where $|A|$ is maximal are called bases of $M$. The literature usually names two standard examples for matroids:

(1) $M=(E, I)$ where $E$ is a finite set of vectors in some $k^{r}, I$ the set of linearly independent subsets of $E$. Clearly rk $M \leq r$.

(2) $M=(E, I)$ where $E$ is the set of edges of a graph and $I$ the set of subgraphs (each determined by a subset of edges) without cycles. Clearly $\operatorname{rk} M=|V(\Gamma)|-\operatorname{rk} H_{0}(\Gamma ; \mathbb{Z})$.

We have already seen in section 2.1 how these examples are related (in fact, the second is a special case of the first): If $\Gamma$ is a graph, for each $e \in E(\Gamma)$ there is a linear form $e^{\vee} j_{\Gamma}$ on $\mathbb{R}^{|V(\Gamma)|} / H_{0}(\Gamma ; \mathbb{R})$, and such linear forms $e_{1}^{\vee} j_{\Gamma}, \ldots, e_{n}^{\vee} j_{\Gamma}$ are pairwise linearly independent if and only if the graph with edges $\left\{e_{1}, \ldots, e_{n}\right\}$ has no cycles.

Let us return to the general case. A matroid is equivalently characterized by a rank function on $2^{E}$ as follows:

Definition 4.2. A map $r: 2^{E} \rightarrow \mathbb{N}$ is called rank function if

(1) $r(A) \leq|A|$

(2) $A_{1} \subseteq A_{2} \Longrightarrow r\left(A_{1}\right) \leq r\left(A_{2}\right)$,

(3) $r\left(A_{1} \cup A_{2}\right)+r\left(A_{1} \cap A_{2}\right) \leq r\left(A_{1}\right)+r\left(A_{2}\right)$.

Proposition 4.1. Let $M=(E, I)$ be a matroid. Then the map

$$
r: A \mapsto \operatorname{rk}(A,\{B \in I, B \subseteq A\})
$$

is a rank function. Conversely, let $E$ be a finite set and $r$ a rank function for it. Then $M=(E, r)=(E, I)$ where $I=\{A \subseteq E, r(A)=|A|\}$ is a matroid.

We have seen how linearly independent subsets of vectors in a vector space give rise to a matroid. On the other hand one may ask if every matroid is obtained this way:

Definition 4.3. Let $k$ be a field. A matroid $M=(E, r)$ is called realizable over $k$ is there is an $r \in \mathbb{N}$ and a map $f: E \rightarrow k^{r}$ with $\operatorname{dim} \operatorname{span} f(A)=r(A)$ for all $A \in 2^{E}$. Such a map is called representation of $M$. 
There are matroids which are representable only over certain fields, for example the Fano matroid.

The space $X(M, s)$ of all representations of $M$ in $k^{s}$ (a subvariety of $\mathbb{A}^{s|E|}$ defined over $k$ ) is called representation space of $M$. It is a fundamental question how general these realization spaces are. An answer is given by Mnëv's Universality Theorem.

Mnëv's Universality Theorem was originally proved by Mnëv in a context of oriented matroids and their representations over the ordered field of real numbers. Without giving a precise definition, an oriented matroid keeps not only track of whether or not certain subsets of vectors are linearly dependent but also about the sign of determinants: Roughly an oriented matroid is specified by a list of partitions of $E$ indicating which vectors in $E$ may be separated by linear hyperplanes in $\mathbb{R}^{n}$. Again the representation space of an oriented matroid is the space of vector configurations which leaves this list of partitions invariant. The original, quite difficult, version of the theorem is then

Theorem 4.1 (Mnëv, oriented version). For every primary semi-algebraic set $X$ in $\mathbb{R}^{r}$ defined over $\mathbb{Z}$ there is an oriented matroid whose realization space is stably equivalent to $X$.

Here a primary semi-algebraic set defined over $\mathbb{Z}$ is a set given by polynomial equations and sharp polynomial inequalities $<$, > with integer coefficients, (such as $x_{1}^{2}+x_{2}^{2}>2, x_{2} x_{1}^{3}=1$ ), and stable equivalence means roughly a sort of homotopy equivalence preserving certain arithmetic properties. The proof in Mnëv's thesis [68, 69] is quite intricate, and there is a simplified proof in [7, 73] which I follow here.

The simpler version that we need is obtained by replacing primary semi-algebraic sets by affine schemes of finite type over $\operatorname{Spec} \mathbb{Z}$, oriented matroids by matroids, and stable equivalent by isomorphic with an open subscheme in a product with $\mathbb{A}^{N}$. Just like the affine representation space, there is a projective representation space

$$
\begin{aligned}
\hat{X}(M, s)= & \left\{f: E \hookrightarrow \mathbb{P}^{s-1}:\right. \\
& \left.\operatorname{dim} \operatorname{span} f(A)=r(A)-1 \text { for all } A \in 2^{E}\right\}
\end{aligned}
$$

Theorem 4.2 (Mnëv, un-oriented version). Let $X$ be an affine scheme of finite type over Spec $\mathbb{Z}$. Then there is a matroid $M$ of rank $3, N \in \mathbb{N}$ and an open $U \subseteq X \times \mathbb{A}^{N}$ projecting surjectively onto $X$ such that

$$
U \cong \hat{X}(M, 3) / P G L_{3} .
$$

This is the version in Lafforgue's book [65]. I am grateful to A. Usnich for showing me this reference. See also [20] for the independently obtained version of Sturmfels. 
Suppose $X$ is defined by $f_{+}-f_{-}=0$ where $f_{+}$and $f_{-}$are polynomials with positive coefficients. The $f_{ \pm}$can be successively decomposed into more elementary expressions involving only one addition or one multiplication at a time, at the expense of introducing many more variables. The proof of Theorem 4.2 uses then the fact that once $x_{1}$ and $x_{2}$ are fixed on a projective line, $x_{1}+x_{2}$ and $x_{1} x_{2}$ etc. can be determined by linear dependence conditions in the projective plane (this is why the rank of $M$ is only 3 ). The difficulties left are to relate different projective scales and to avoid unwanted dependencies.

Like this any affine scheme over $\operatorname{Spec} \mathbb{Z}$ is related to the representation space of a (huge) rank 3 matroid. Belkale and Brosnan use a slightly different version of Mnëv's theorem and then show (a lot of work that I just skip) how this representation space is connected to the graph hypersurfaces $C X_{\Gamma}$.

Let me now state the main result of [7]: Let $\mathrm{GeoMot}^{+}$be the abelian group with generators isomorphism classes $[X]$ of schemes $X$ of finite type over $\mathbb{Z}$ modulo the relation

$$
[X]=[X \backslash V]+[V]
$$

if $V$ is a closed subscheme of $X$. Endowed with the cartesian product $[X][Y]=$ $[X \times Y]$, GeoMot ${ }^{+}$becomes a ring with unit $[\operatorname{Spec} \mathbb{Z}]$. Let $L=\left[\mathbb{A}^{1}\right]$ be the Tate motive, and $S$ the saturated multiplicative subset of $\mathbb{Z}[L]$ generated by $L^{n}-L$ for $n>1$. Let GeoMot $=S^{-1}$ GeoMot $^{+}$, and Graphs the $S^{-1} \mathbb{Z}[L]$-submodule of GeoMot generated by the $\left[C X_{\Gamma}\right]$, where $\Gamma$ are Feynman graphs.

Theorem 4.3 (Belkale, Brosnan). Graphs = GeoMot .

It is clear that point-counting $q \mapsto\left|X\left(\mathbb{F}_{q}\right)\right|$ factors through GeoMot. Therefore Kontsevich's conjecture is false. Also it is known [7, Section 15] that the mixed Tate property can be detected in GeoMot. Therefore it follows that not all $X_{\Gamma}$ are mixed Tate, and (using another conjecture) that not all periods of all $X_{\Gamma}$ are rational linear combinations of multiple zeta values.

On the other hand, not all periods of all $X_{\Gamma}$ are Feynman-periods in the sense defined in section 4.2 .

4.4. The work of Bloch, Esnault and Kreimer. A finer study of motives of certain Feynman graph hypersurfaces is carried out in the second part of [15]: For the so called wheels with $n$ spokes,

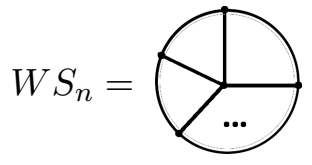

one has

Theorem 4.4 (Bloch, Esnault, Kreimer).

$$
H_{c}^{2 n-1}\left(\mathbb{P}^{2 n-1} \backslash X_{W S_{n}}\right) \cong \mathbb{Q}(-2), H^{2 n-1}\left(\mathbb{P}^{2 n-1} \backslash X_{W S_{n}}\right) \cong \mathbb{Q}(-2 n+3)
$$


and $H_{d R}^{2 n-1}\left(\mathbb{P}^{2 n-1} \backslash X_{W S_{n}}\right)$ is generated by $\Omega / \Psi_{W S_{n}}^{2}$.

It had been known before [21,22] that

$$
\operatorname{res}_{S} W S_{n} \in \zeta(2 n-3) \mathbb{Q}^{\times}
$$

and Theorem 4.4 partially confirms that an extension

$$
0 \rightarrow \mathbb{Q}(2 n-3) \rightarrow E \rightarrow \mathbb{Q}(0) \rightarrow 0
$$

is responsible for this (see [15, Section 9],[14, Section 9]).

4.5. The work of Bloch and Kreimer on renormalization. Let us return to renormalization. Within the parametric Feynman rules, Bloch and Kreimer [16] show how to understand renormalized non-primitive integrals using periods of a limiting mixed Hodge structure.

Limiting mixed Hodge structures arise in a situation where there is a family of Hodge structures varying over a base space, in this case a punctured disk $D^{*}$ (For zero momentum transfer Feynman graphs this one-dimensional base space is sufficient). In contrast to section 3.4 the parameter $t \in D^{*}$ does not alter the exponent of the differential form, but is rather some sort of cut-off for the chain of integration.

It follows from our discussion in 3.4 that the projective integral

$$
\int_{\sigma} \frac{\Omega}{\Psi_{\Gamma}^{2}}
$$

is not convergent unless $\Gamma$ is primitive (This is the reason why $\operatorname{res}_{S} \Gamma$ is defined only for primitive integrals): there are poles along the exceptional divisors $\mathcal{E}_{L_{\gamma}}$ corresponding to divergent subgraphs $\gamma$. In other words, $\int_{\sigma} \frac{\Omega}{\Psi_{\Gamma}^{2}}$ is not a period. But by varying the coordinate divisor $\Delta_{t}$ (and the simplex $\sigma_{t}$ sitting inside $\Delta_{t}$ ) with $t \in D^{*}$, one has a family of mixed Hodge structures, and for all $t \neq 0$ the period $\int_{\sigma_{t}} \frac{\Omega}{\Psi_{\Gamma}^{2}}$ is defined.

Bloch and Kreimer describe how to express the monodromy operation on (relative) homology, in particular on $\sigma_{t}$, in terms of suitable tubes around the strata of the exceptional divisor of $\mathcal{Z}_{S}$. Winding around such a tube picks up the residue along the stratum (see section 3.4). Since the monodromy is quasi-unipotent, its logarithm gives a (graph-independent) nilpotent matrix $N$ such that

$$
\int_{\sigma_{t}} \frac{\Omega}{\Psi_{\Gamma}^{2}}=\text { first row of } \exp (N \log t / 2 \pi i)\left(a_{1}, \ldots, a_{r}\right)^{t}
$$

up to a multi-valued analytic function vanishing at $t=0$, with $a_{1}, \ldots, a_{r}$ periods of a limiting mixed Hodge structure [16].

When there is only one non-zero external momentum, say $P$, the relation between the regularization (27) and the renormalized integral (19), where the second graph polynomial must be taken into account, is easy to see. Therefore (27) also 
tells about the coefficients $p_{n}(\Gamma)$ of the renormalized integral (19), and one observes in the monodromy representation the same combinatorial objects (nested sets, the Connes-Kreimer coproduct) that have guaranteed locality of counterterms in section 3 .

4.6. Final remarks. Let me finish this second part of the paper by just mentioning very briefly some other results that have been obtained in this area.

The Belkale-Brosnan theorem does not provide a specific counterexample graph to Kontsevich's conjecture (it does provide a counterexample matroid). See [35,75] for recent developments in this direction.

The methods of [15] have been extended in [36] to other graphs than the wheels with spokes. Regularization and renormalization in the parametric representation is also discussed in [17, 18, 66].

The relation between Feynman periods and multiple zeta values as periods of the moduli space of stable genus 0 curves is studied much further in [24,25]. Finally the reader may be interested in [1,-3, 13, 71] for a further study of graph hypersurfaces.

\section{REFERENCES}

[1] P. Aluffi and M. Marcolli, Algebro-geometric Feynman rules. arXiv:0811.2514.

[2] P. Aluffi and M. Marcolli, Feynman motives and deletion-contraction relations. arXiv:0907.3225.

[3] P. Aluffi and M. Marcolli, Parametric Feynman integrals and determinant hypersurfaces. arXiv:0901.2107.

[4] Y. André, Une introduction aux motifs (Motifs purs, motifs mixtes, périodes), Panoramas et Synthéses 17, SMF, 2004.

[5] M. Atiyah, Resolution of singularities and divison of distributions, Comm. Pure Appl. Math. XXIII (1970), 145-150.

[6] S. Axelrod and I. M. Singer, Chern-Simons perturbation theory 2, J. Diff. Geom. 39 (1994), 173-213. hep-th/9304087.

[7] P. Belkale and P. Brosnan, Matroids, motives, and a conjecture of Kontsevich, Duke Math. J. 116 (2003), no. 1, 147-188. math.AG/0012198.

[8] P. Belkale and P. Brosnan, Periods and Igusa local zeta functions, Int. Math. Res. Not. 49 (2003), 2655-2670. math/0302090.

[9] C. Bergbauer, Combinatorial and geometric aspects of Feynman graphs and Feynman integrals. PhD thesis, Freie Universität Berlin, April 2009. Published online at http://www.diss.fuberlin.de/diss/receive/FUDISS_thesis_000000010972.

[10] C. Bergbauer, R. Brunetti, and D. Kreimer, Renormalization and resolution of singularities. arXiv:0908.0633.

[11] C. Bergbauer and D. Kreimer, Hopf algebras in renormalization theory: Locality and DysonSchwinger equations from Hochschild cohomology, IRMA Lect. Math. Theor. Phys. 10 (2006), 133-164. hep-th/0506190.

[12] I. N. Bernstein and S. I. Gel'fand, Meromorphy of the function $P^{\lambda}$, Funkcional. Anal. i Priložen. 3 (1969), no. 1, 84-85.

[13] S. Bloch, Motives associated to sums of graphs. arXiv:0810.1313.

[14] S. Bloch, Motives associated to graphs, Jpn. J. Math. 2 (2007), no. 1, 165-196.

[15] S. Bloch, H. Esnault, and D. Kreimer, On Motives Associated to Graph Polynomials, Commun. Math. Phys. 267 (2006), 181-225. math.AG/0510011. 
[16] S. Bloch and D. Kreimer, Mixed Hodge structures and renormalization in phyics, Commun. Number Theory Phys. 2 (2008), no. 4, 637-718. arXiv:0804.4399.

[17] C. Bogner and S. Weinzierl, Resolution of singularities for multi-loop integrals, Comput. Phys. Commun. 178 (2008), 596-610. arXiv:0709.4092.

[18] C. Bogner and S. Weinzierl, Periods and Feynman integrals, J. Math. Phys. 50 (2009), 042302. arXiv:0711.4863.

[19] N. N. Bogolyubov and D. V. Shirkov, Introduction to the theory of quantized fields, 3rd ed., Wiley, 1980.

[20] J. Bokowski and B. Sturmfels, Computational synthetic geometry, Lecture Notes in Mathematics, vol. 1355, Springer-Verlag, Berlin, 1989.

[21] D. Broadhurst and D. Kreimer, Knots and numbers in $\Phi^{4}$ theory to 7 loops and beyond, Int. J. Mod. Phys. C6 (1995), 519-524. hep-ph/9504352.

[22] D. Broadhurst and D. Kreimer, Association of multiple zeta values with positive knots via feynman diagrams up to 9 loops, Phys. Lett. B393 (1997), 403-412. hep-th/9609128.

[23] D. Broadhurst and D. Kreimer, Exact solutions of Dyson-Schwinger equations for iterated one-loop integrals and propagator-coupling duality, Nucl. Phys. B600 (2001), 403-422. hepth/0012146.

[24] F. Brown, On the periods of some Feynman integrals. arXiv:0910.0114.

[25] F. Brown, The massless higher-loop two-point function, Commun. Math. Phys. 287 (2009), 925-958. arXiv:0804.1660.

[26] F. C. S. Brown, Multiple zeta values and periods of moduli spaces $\overline{\mathfrak{M}}_{0, n}$, Ann. Sci. Éc. Norm. Supér. (4) 42 (2009), no. 3, 371-489.

[27] J. Carlson, S. Müller-Stach, and C. Peters, Period mappings and period domains, Cambridge Studies in Advanced Mathematics, vol. 85, Cambridge University Press, Cambridge, 2003.

[28] J. Collins, Renormalization, Cambridge Monographs on Mathematical Physics, Cambridge University Press, 1984.

[29] A. Connes and D. Kreimer, Hopf algebras, renormalization and noncommutative geometry, Commun. Math. Phys. 199 (1998), 203-242. hep-th/9808042.

[30] A. Connes and D. Kreimer, Renormalization in quantum field theory and the Riemann- Hilbert problem I: The Hopf algebra structure of graphs and the main theorem, Comm. Math. Phys. 210 (2000), 249-273. hep-th/9912092.

[31] A. Connes and D. Kreimer, Renormalization in quantum field theory and the RiemannHilbert problem II: The beta-function, diffeomorphisms and the renormalization group, Commun. Math. Phys. 216 (2001), 215-241. hep-th/0003188.

[32] A. Connes and M. Marcolli, Noncommutative Geometry, Quantum Fields, and Motives (2007).

[33] P. Cvitanovic, Field theory, Nordita, 1983.

[34] C. De Concini and C. Procesi, Wonderful models of subspace arrangements, Selecta Math. (N. S. ) 1 (1995), no. 3, 459-494.

[35] D. Doryn. Talk given at the IHES, June 2009.

[36] D. Doryn, Cohomology of graph hypersurfaces associated to certain Feynman graphs. Thesis (2008), Universität Duisburg-Essen. arXiv:0811.0402.

[37] K. Ebrahimi-Fard, L. Guo, and D. Kreimer, Spitzer's identity and the algebraic Birkhoff decomposition in pQFT, J. Phys. A37 (2004), 11037-11052. hep-th/0407082.

[38] H. Epstein and V. Glaser, The role of locality in perturbation theory, Annales Poincare Phys. Theor. A19 (1973), 211.

[39] P. Etingof, Note on dimensional regularization, Quantum fields and strings: a course for mathematicians, Vol. 1, 2 (Princeton, NJ, 1996/1997), 1999, pp. 597-607.

[40] L. Foissy, Systems of Dyson-Schwinger equations. arXiv:0909.0358.

[41] L. Foissy, Les algèbres de Hopf des arbres enracinés I-II, Bull. Sci. Math. 126 (2002), 193-239 and 249-288.

[42] K. Fredenhagen, Quantenfeldtheorie I, II; Quantenfeldtheorie auf gekrümmter Raumzeit, University of Hamburg, http://www.desy.de/uni-th/lqp/notes.html. 
[43] W. Fulton and R. MacPherson, A compactification of configuration spaces, Ann. Math. 139 (1994), 183-225.

[44] A. Goncharov and Y. Manin, Multiple $\zeta$-motives and moduli spaces $\overline{\mathcal{M}}_{0, n}$, Compos. Math. 140 (2004), no. 1, 1-14. math.AG/0204102.

[45] R. Haag, Local quantum physics, fields, particles, algebras, 2nd ed., Springer, Berlin, 1996.

[46] K. Hepp, Proof of the Bogolyubov-Parasiuk theorem on renormalization, Commun. Math. Phys. 2 (1966), 301-326.

[47] C. Itzykson and J. -B. Zuber, Quantum field theory, McGraw-Hill, 1980.

[48] D. Kazhdan, Introduction to QFT, Quantum fields and strings: a course for mathematicians, Vol. 1, 2 (Princeton, NJ, 1996/1997), 1999, pp. 377-418.

[49] M. Kontsevich. Talk given at the IHES, 2003.

[50] M. Kontsevich. Talk given at the Gelfand seminar, Rutgers, 1997.

[51] M. Kontsevich, Feynman diagrams and low-dimensional topology, First European Congress of Mathematics, Vol. II (Paris, 1992), 1994, pp. 97-121.

[52] M. Kontsevich and D. Zagier, Periods, Mathematics Unlimited - 2001 and Beyond, 2001, pp. 771-808.

[53] D. Kreimer, On the Hopf algebra structure of perturbative quantum field theories, Adv. Theor. Math. Phys. 2 (1998), 303-334. q-alg/9707029.

[54] D. Kreimer, Knots and Feynman diagrams, Cambridge University Press, 2000.

[55] D. Kreimer, Anatomy of a gauge theory, Annals Phys. 321 (2006), 2757-2781.

[56] D. Kreimer, The residues of quantum field theory: Numbers we should know, Noncommutative Geometry and Number Theory (bonn, 2003), 2006, pp. 187-204. hep-th/0404090.

[57] D. Kreimer, Dyson-Schwinger equations: from Hopf algebras to number theory, Universality and renormalization, 2007, pp. 225-248.

[58] D. Kreimer, Factorization in quantum field theory: An exercise in Hopf algebras and local singularities, Frontiers in number theory, physics, and geometry. II, 2007, pp. 715-736. hepth/0306020.

[59] D. Kreimer, A remark on quantum gravity, Annals Phys. 323 (2008), 49-60. arXiv:0705.3897.

[60] D. Kreimer, Étude for linear Dyson-Schwinger equations, Traces in number theory, geometry and quantum fields, 2008, pp. 155-160.

[61] D. Kreimer and W. D. van Suijlekom, Recursive relations in the core Hopf algebra. arXiv:0903.2849.

[62] D. Kreimer and K. Yeats, An etude in non-linear Dyson-Schwinger equations, Nucl. Phys. Proc. Suppl. 160 (2006), 116-121. hep-th/0605096.

[63] D. Kreimer and K. Yeats, Recursion and growth estimates in renormalizable quantum field theory, Commun. Math. Phys. 279 (2008), no. 2, 401-427. hep-th/0612179.

[64] K. Kremnizer and M. Szczesny, Feynman graphs, rooted trees, and Ringel-Hall algebras, Comm. Math. Phys. 289 (2009), no. 2, 561-577.

[65] L. Lafforgue, Chirugie des grassmanniennes, CRM Monograph Series, vol. 19, AMS, Providence, RI, 2003.

[66] M. Marcolli, Motivic renormalization and singularities. arXiv:0804.4824.

[67] I. Mencattini and D. Kreimer, The structure of the Ladder Insertion-Elimination Lie algebra, Commun. Math. Phys. 259 (2005), 413-432. math-ph/0408053.

[68] N. E. Mnëv, Varieties of combinatorial types of projective configurations and convex polyhedra, Dokl. Akad. Nauk SSSR 283 (1985), no. 6, 1312-1314.

[69] N. E. Mnëv, The universality theorems on the classification problem of configuration varieties and convex polytopes varieties, Topology and geometry-Rohlin Seminar, 1988, pp. 527-543.

[70] K. Osterwalder and R. Schrader, Axioms for Euclidean Green's functions I and II, Commun. Math. Phys. 31 (1973), 83-112. and 42 (1975) 281.

[71] E. Patterson, On the singular structure of graph hypersurfaces. arXiv:1004.5166.

[72] M. Reed and B. Simon, Fourier analysis, self-adjointness, Methods of modern mathematical physics, vol. 2, Academic Press, New York, 1975. 
[73] J. Richter-Gebert, Mnëv's universality theorem revisited, Sém. Lothar. Combin. 34 (1995), Art. B34h, approx. $15 \mathrm{pp}$. (electronic).

[74] V. Rivasseau, From Perturbative to Constructive Renormalization, Princeton University Press, 1991.

[75] O. Schnetz, Quantum field theory over $F_{q}$. arXiv:0909.0905.

[76] O. Schnetz, Quantum periods: A census of $\phi^{4}$-transcendentals. arXiv:0801.2856.

[77] R. F. Streater and A. S. Wightman, PCT, Spin and Statistics, and All That, Princeton, reprint 2000.

[78] W. van Suijlekom, The Hopf algebra of Feynman graphs in QED, Lett. Math. Phys. 77 (2006), 265-281. hep-th/0602126.

[79] M. Szczesny, On the structure and representations of the insertion-elimination Lie algebra, Lett. Math. Phys. 84 (2008), no. 1, 65-74.

[80] M. Szczesny, Hecke correspondences and Feynman graphs, Comm. Number Theory and Phys. 4 (2010).

[81] A. Usnich. Private communication.

[82] G. van Baalen, D. Kreimer, D. Uminsky, and K. Yeats, The QED beta-function from global solutions to Dyson- Schwinger equations, Ann. Phys 324 (2009), 205-219. arXiv:0805.0826.

[83] G. van Baalen, D. Kreimer, D. Uminsky, and K. Yeats, The QCD beta-function from global solutions to Dyson- Schwinger equations, Ann. Phys. 325 (2010), 300-324. arXiv:0906.1754.

[84] W. van Suijlekom, Multiplicative renormalization and Hopf algebras. arXiv:0707.0555.

[85] W. D. van Suijlekom, Renormalization of gauge fields: A Hopf algebra approach, Commun. Math. Phys. 276 (2007), 773-798. hep-th/0610137.

[86] M. J. G. Veltman, Diagrammatica: The Path to Feynman rules. Cambridge, UK: Univ. Pr. (1994) 284 p. (Cambridge lecture notes in physics, 4).

[87] S. Weinberg, The Quantum Theory of Fields I, Cambridge University Press, 1985.

[88] K. Yeats, Growth estimates for Dyson-Schwinger equations. Thesis, Boston University (2008). arXiv:0810.2249.

[89] W. Zimmermann, Convergence of Bogolyubov's method of renormalization in momentum space, Commun. Math. Phys. 15 (1969), 208-234.

SFB 45, Institut FÜR MATHEMATIK, JohANNES-GUtenBERG-UNIVERSitÄt MAINZ

E-mail address: bergbau@math. fu-berlin.de 